\title{
GENERAL THEORY OF LINEAR DIFFERENCE EQUATIONS*
}

\author{
BY
}

\section{GEORGE D. BIRKHOFF}

\section{INTRODUCTION.}

The theory of linear difference equations with rational coefficients was in a very backward state until PoINCARE $\dagger$ in 1882 developed the notion of asymptotic representation, and its application to this branch of mathematics. Further important progress in this direction has been made only very recently, notably by GALBRUN, $\ddagger$ who by means of the Laplace transformation has investigated the properties of a set of fundamental solutions in the entire plane of the complex variable $x$.

The ain of the present paper is first to study the nature of these solutions to which I have been led by means of direct methods, and secondly to show that there exists a purely Riemannian theory of linear difference equations. In particular certain rational functions of $e^{2 r^{\prime}-1} x$ are shown to play a part like that of the monodromic group constants of an ordinary linear differential equation.

To the best of my knowledge, the importance of the functional standpoint in the field of difference equations was emphasized first by VAN VLECK in an inspiring series of lectures given at the University of Wisconsin in the spring of 1909 , in which he conjectured the existence of sets of solutions analytic on either the left or the right side of the complex plane.

On account of the extreme simplicity of the matrix notation, I have found it convenient to deal with a linear difference system of $n$ equations of the first order

$$
g_{i}(x+1)=\sum_{j=1}^{n} a_{i j}(x) g_{j}(x), \quad(i=1,2, \cdots, n),
$$

rather than with a single equation of the $n$th order. In these equations the functions $a_{i j}(x)$ are taken to be rational functions of $x$ with a pole at $x=\infty$ of order $\mu$ at most, so that one has

$$
a_{i j}(x)=a_{i j} x^{\mu}+a_{i j}^{(1)} x^{\mu-1}+\cdots, \quad(|x|>R) .
$$

* Presented to the Society, September 6, 1910.

+ American Journal of Mathematics, vol. 7 (1885), pp. 203-258.

$¥$ Comptes Rendus, vol. 148 (1909), pp. 905-907. 
The sole other restriction to be imposed on these functions below will, in particular, be satisfied if the determinantal characteristic equation

$$
\left|a_{i j}-\delta_{i j} \rho_{j}\right|=0
$$

[where $\delta_{i j}=0, i \neq j ; \delta_{i i}=1$ ] has $n$ distinct roots which are not zero. If the $n$ sets of functions

$$
\begin{gathered}
g_{11}(x), \cdots, g_{n 1}(x) \\
\cdot \cdot \cdot \cdot \cdot \cdot \\
g_{1 n}(x), \cdots, g_{n n}(x)
\end{gathered}
$$

are $n$ linearly independent solutions of (1), the array of elements $g_{i v}(x)$ forms a matrix solution $G(x)$. The array of elements $a_{i j}(x)$ forms a second matrix $A(x)$, and the $n^{2}$ equations which the $n$ solutions satisfy can be combined into a single matrix equation

$$
G(x+1)=A(x) G(x) .
$$

If $x$ be replaced by $x-1$, this may be written

$$
G(x-1)=A^{-1}(x-1) G(x) \text {. }
$$

The most general matrix solution of $(4)$ is furnished by

$$
H(x)=G(x) P(x),
$$

in which $G(x)$ is any particular matrix solution, and $P(x)$ is any arbitrary matrix of periodic functions of period 1 whose determinant does not vanish identically.

The equation (4) admits of two symbolic solutions

$$
\left\{\begin{array}{l}
G(x)=A(x-1) A(x-2) \cdots \\
G(x)=A^{-1}(x) A^{-1}(x+1) \cdots
\end{array}\right.
$$

which converge to limit matrices only in particular cases. This convergence is secured to a sufficient extent by a suitable modification, made in $\$ 1$, as follows: The system (1) is known to be satisfied formally by taking for $g_{1}(x), g_{2}(x)$, $\cdots, g_{n}(x)$ certain formal series, one set of them for each of the $n$ roots $\rho$ of the characteristic equation (3). The matrix formed from these sets taken in a certain order is denoted by $S(x)$, and the modified matrix obtained by breaking off these elements at the $k$ th term is called $T(x)$. The sequences of matrices

$$
\begin{aligned}
& A(x-1) A(x-2) \cdots A(x-m) T(x-m), \\
& A^{-1}(x) A^{-1}(x+1) \cdots A^{-1}(x+m-1) T(x+m), \quad(m=1,2, \cdots),
\end{aligned}
$$

form the desired modification of the two symbolic solutions. It is demonstrated that all the determinants formed from the first $\lambda$ columns and any $\lambda$ rows of the 
first of these matrices, $(\lambda=1,2, \cdots, n)$, or from the last $\lambda$ columns and any $\lambda$ rows of the second of these matrices, converge to limit functions independent of $k$ as $m$ becomes infinite. By the aid of these functions and by a process of summation based on a contour integral suggested by the one which CARMICHAEL * has used, two remarkable particular matrix solutions $G(x)$ and $H(x)$ are obtained in $\$ \S 2,3,4$. These are termed the first and second principal matrix solutions respectively. The elements of $G(x)$ are analytic, save for poles at points $\sigma+1, \sigma+2, \ldots$, where $\sigma$ is a pole of one of the elements of $A(x)$, and have the asymptotic form of the corresponding elements of $S(x)$ in any left half plane. The elements of $H(x)$ are analytic save for poles at points $\sigma-1, \sigma-2, \cdots$, where $\sigma$ is a pole of one of the elements of $A^{-1}(x-1)$, and have the asymptotic form of the corresponding elements of $S(x)$ in any right half plane. The principal matrix solutions $G(x)$ and $H(x)$ are uniquely determined by this asymptotic property. A relation (5) will also, of course, subsist between $G(x)$ and $H(x)$.

Conversely if $G(x)$ and $H(x)$ are asymptotically represented by the elements of a matrix $S(x)$ even to terms of the first order and are related by means of a matrix $P(x)$ of periodic functions, as in (5), these matrices $G(x)$ and $H(x)$ will form the first and second principal matrix solutions of a system (4) in which the elements of $A(x)$ are rational (see $\S 7$ ).

It is proved in $\S 5$ that the elements of $P(x)$ are rational functions of $e^{2 \pi^{2}-1 x}$. The structure of these functions is determined with particular reference to the case when the functions $a_{i j}(x)$ are polynomials of degree $\mu$, to which case the more general one is reduced.

These results lead in $\$ 6$ to the determination of the nature of the elements of $G(x)$ and $H(x)$ in the remaining right and left half plane respectively; the asymptotic form is found to alter along certain critical rays. $\mathbf{A}$ complete description of the principal matrix solutions is thus obtained.

In $\S 7$ it is shown that the number of characteristic constants involved in the complete characterization of $G(x)$ and $H(x)$ and their connection by means of $P(x)$ is precisely equal to the number of arbitrary constants in the coefficients $a_{i j}(x)$ assumed to be polynomials, and that the characteristic constants are independent. Thus $I$ have ventured to propose the following as a fundamental problem of linear difference equations : to determine whether there exists a linear difference system with prescribed characteristic constants.

It would be interesting to know the exact relation between the characteristic constants and the monodromic group constants of the differential equation yielded by the Laplace transformation.

The method contained in the present paper is of wide generality and applies if the coefficients $a_{i j}(x)$ have the character of rational functions only at $x=\infty$,

* These Transactions, vol. 12 (1911), pp. 99-134. 
in all cases where there exist $n$ formal solutions. Carmichael (loc. cit.) has studied this case to a considerable extent; in particular he has proved the existence of analytic solutions and has partly discussed their asymptotic form in the direction of the axis of reals.

\section{§1. The Determinant Limits.}

It is apparent that the given linear system (1), when written in the matrix notation (4), (4)', possesses the two symbolic solutions

$$
A(x-1) A(x-2) \cdots, \quad A^{-1}(x) A^{-1}(x+1) \cdots
$$

These will converge to limit matrices in certain special cases, for example if $a_{i j}(x)$ has the form

$$
\delta_{i j}+\frac{a_{i j}^{(2)}}{x^{2}}+\frac{a_{i j}^{(3)}}{x^{3}}+\cdots ;
$$

in this case the above symbolic solutions will yield the complete solution of (1). In general however these expressions must be properly modified in order to be made to lead indirectly to a complete solution of (1).

Let us first make precise the limitation which is to be made on the coefficients $a_{i j}(x)$ in equations (1). If the roots of the characteristic equation (3) are distinct, and if none of them are zero, there will exist precisely $n$ sets of series

$$
\begin{aligned}
& s_{1 j}(x)=x^{\mu x}\left(\rho_{j} e^{-\mu}\right)^{x} x^{r_{j}}\left\{s_{1 j}+\frac{s_{1 j}^{(1)}}{x}+\cdots\right\}, \\
& s_{2 j}(x)=x^{\mu x} \cdot\left(\rho_{j} e^{-\mu}\right)^{x} x^{r_{j}}\left\{s_{2 j}+\frac{s_{2 j}^{(1)}}{x}+\cdots\right\}, \quad(j=1,2, \cdots, n), \\
& s_{n j}(x)=x^{\mu x}\left(\rho_{j} e^{-\mu}\right)^{x} x^{r_{j}}\left\{s_{n j}+\frac{s_{n j}^{(1)}}{x}+\cdots\right\},
\end{aligned}
$$

each of which constitutes a formal solution of (1). The proof of this is a matter of direct reckoning, and the fact will be taken for granted.* The constants $\rho_{1}, \rho_{2}, \cdots, \rho_{n}$ are the roots of the characteristic equation, and furthermore we have the determinant

$$
d=\left|s_{i j}\right| \neq 0 \text {. }
$$

* The formal solutions of a single linear difference equation of the $n$th order have been frequently used (for example see HoRN, Crelle's Journal, vol. 138 (1910), pp. 159-191) and admit of easy extension to a linear system. The formal solutions may be obtained by direct substitution if the roots of the characteristic equation are distinct and different from zero; the series expansion

$$
\left(1+\frac{1}{x}\right)^{\mu x}=e^{\mu x \log \left(1+\frac{1}{x}\right)}=\epsilon^{\mu}-\frac{\mu e^{\mu}}{2 x}+\cdots
$$

enables one to make a comparison of coefficients and thus to determine the constants of the formal solutions. 
The restrictive hypothesis which we shall make is not that $\rho_{1}, \rho_{2}, \cdots, \rho_{n}$ are distinct but only that there exist $n$, formal solutions (6) with the specified property (7). The quantities $\rho_{1}, \rho_{2}, \ldots, \rho_{n}$ must of course still be the roots of the characteristic equation, and none of them can be zero. We shall choose our subscripts so that

$$
\left|\rho_{1}\right| \geqq\left|\rho_{2}\right| \geqq \ldots \geqq\left|\rho_{n}\right| \text {. }
$$

Without loss of real generality we may add the further condition that the linear homogeneous difference equation of the $n$th order at most which $g_{1}(x)$ satisfies, and which is obtained from (1) by elimination, is actually of the $n$th order.

The elements $s_{i j}(x)$ form a matrix $S(x)$ which is a formal matrix solution of the equation (4)

$$
S(x+1)=A(x) S(x) \text {. }
$$

Let us compute the elements $s_{i j}^{\prime}(x)$ of the inverse matrix $S^{-1}(x)$. The determinant $|S(x)|$ may be written

$$
x^{n \mu x}\left(\rho_{1} \rho_{2} \cdots \rho_{n} e^{-n \mu}\right)^{x} x^{r_{1}+r_{2}+\cdots+r_{n}}\left\{d+\frac{d^{(1)}}{x}+\cdots\right\} .
$$

The element in the $i$ th row and $j$ th column of the inverse matrix is the quotient of the cofactor of the element in the $j$ th row and the $i$ th column of this determinant by the determinant itself, and is therefore given by a formal series

$$
s_{i j}^{\prime}(x)=x^{-\mu x}\left(\rho_{i} e^{-\mu}\right)^{-x} x^{-r_{i}}\left\{\sigma_{i j}+\frac{\sigma_{i j}^{(1)}}{x}+\cdots\right\} .
$$

Let $T(x)$ denote any matrix obtained by breaking off the elements of $S(x)$ at the $k$ th term, or more generally by replacing $s_{i j}(x)$ by convergent series $t_{i j}(x)$ of the same form as $s_{i j}(x)$ and having the same first $k$ terms. W rite then

$$
T(x+1)=B(x) T(x) .
$$

The matrix $B(x)$, as thus defined, is clearly a matrix of functions $b_{i j}(x)$ each of which has an expansion like $a_{i j}(x)$ at $x=\infty$ with precisely the same first $k$ terms. For if we compare the formulas

$$
B(x)=T(x+1) T^{-1}(x) \quad \text { and } \quad A(x)=S(x+1) S^{-1}(x),
$$

we observe first that the element $t_{i j}^{\prime}(x)$ of $T^{-1}(x)$ is given by $(9)$ to the first $k$ terms, and that in consequence the right hand members of both the equations have the same first $k$ terms. In consequence we may put

$$
A(x)=B(x)\left[1+x^{-k} C(x)\right],
$$

1 being the identity matrix, and the elements of $C(x)$ being analytic at $x=\infty$. It is to be noted that the determinant formed from the principal coefficients of 
$A(x)$ and $B(x)$, namely $\left|a_{i j}\right|$, cannot be zero, for that implies that zero is a root of the characteristic equation.

In this paper it is necessary to distinguish sharply between two kinds of asymptotic representation of a function by a series of the form (6), say

$$
s(x)=x^{\mu x}\left(\rho e^{-\mu}\right)^{x} x^{r}\left(s+\frac{s^{(1)}}{x}+\cdots\right) .
$$

Let $g(x)$ be the given function; if for each $k$ the difference

$$
g(x) x^{-\mu x}\left(\rho e^{-\mu}\right)^{-x} x^{-r}-\left(s+\frac{s^{(1)}}{x}+\cdots+\frac{s^{(k)}}{x^{k}}\right)
$$

becomes uniformly small of order $x^{-k}$ for $\lim x=\infty$ in a certain region, we say that $g(x)$ is asymptotically represented by $s(x)$ in that region, with respect to $x$; if on the other hand the difference is uniformly small of order $v^{-k}$ (where $x=u+\sqrt{-1} v, u, v$ being real quantities) for $\lim v=\infty$ in the region, we say that the representation is with respect to $v$. In this connection two important inequalities are

$$
\sum_{\nu=1}^{\infty}\left|\frac{1}{(x-\nu)^{k}}\right|<\frac{\pi}{2|x|^{k-1}}
$$

for $x$ in the left half of the complex plane, and

$$
\sum_{\nu=1}^{\infty}\left|\frac{1}{(x-\nu)^{k}}\right|<\frac{2}{|v|^{k-1}}\left[\frac{1}{|v|}+\frac{\pi}{2}\right]
$$

for $x$ in the right half of the complex plane. To prove the first of these we note that when $x$ lies in the left half plane $u$ is negative, so that

$$
|x-\nu|^{2}=(u-\nu)^{2}+v^{2}>u^{2}+v^{2}+\nu^{2},
$$

whence the sum of the given series is less than

$$
\sum_{\nu=1}^{\infty} \frac{1}{\left(|x|^{2}+\nu^{2}\right)^{\frac{1}{2} k}}<\int_{0}^{\infty} \frac{1}{|x|^{k-2}} \frac{d \nu}{\left(|x|^{2}+\nu^{2}\right)}=\frac{\pi}{2|x|^{k-1}} .
$$

Again, for $x$ in the right half plane, our series may be written

$$
\sum_{\nu=1}^{\infty} \frac{1}{\left[(u-\nu)^{2}+v^{2}\right]^{1 k}}<\sum_{\nu=-\infty}^{+\infty} \frac{1}{\left[(u-\nu)^{2}+v^{2}\right]^{\frac{1 k}{k}}}<\frac{1}{|v|^{k-2}} \sum_{\nu=-\infty}^{+\infty} \frac{1}{(u-\nu)^{2}+v^{2}} .
$$

If each quantity $|u-\nu|$ be replaced by the last of the integers $0,1,2, \ldots$ which does not exceed it, the terms in the last series are not decreased, and hence one has

$$
\frac{1}{|v|^{k-2}} \sum_{\nu=-\infty}^{+\infty} \frac{1}{(u-\nu)^{2}+v^{2}} \leqq \frac{2}{|v|^{k-2}} \sum_{\nu=0}^{\infty} \frac{1}{\nu^{2}+v^{2}}<\frac{2}{|v|^{k-2}}\left[\frac{1}{v^{2}}+\int_{0}^{\infty} \frac{d \nu}{\nu^{2}+v^{2}}\right] .
$$


But the last integral reduces to $\pi / 2|v|$, and thus the second inequality holds also.

We may now proceed to the existence theorem :

Theorem I. Form the two sequences of matrices

$$
\begin{aligned}
& P_{m}(x)=A(x-1) A(x-2) \cdots A(x-m) T(x-m), \\
& Q_{m}(x)=A^{-1}(x) A^{-1}(x+1) \cdots A^{-1}(x+m-1) T(x+m)
\end{aligned}(m=1,2, \cdots) .
$$

Every determinant formed from the first $\lambda$ columns $(\lambda=1,2, \ldots, n)$ and the ith, jth, ..., lth rows of $\boldsymbol{P}_{m}(x)\left[Q_{m}(x)\right]$ converges, for $k$ sufficiently large, to a definite limit function $u_{i j \ldots l}(x)\left[v_{i j \ldots l}(x)\right]$, independent of $k$, as $m$ becomes infinite. This function is analytic throughout the entire x-plane excepting at points any number of units to the right (left) of poles of the elements of $A(x)\left[A^{-1}(x-1)\right]$ and at these points may have a pole. The asymptotic form of $u_{i j \ldots l}(x)\left[v_{i j \ldots l}(x)\right]$ in the vicinity of $x=\infty$ is given by the corresponding determinant $s_{i j} \ldots l(x)$ of $S(x)$, with respect to $x$ in any left (right) half of the plane, and with respect to $v$ in any right (left) half of the plane.

Proof. We shall first prove the theorem in so far as it relates to the case $\lambda=1$, and then show how the cases $\lambda=2,3, \cdots, n$ may be reduced to this case.

It is necessary first to show that the elements of the first column of $\boldsymbol{P}_{\mathrm{m}}(x)$ converge to definite limit functions $u_{1}(x), u_{2}(x), \cdots, u_{n}(x)$ as $m$ becomes infinite.

The matrix $P_{m}(x)$ may be written

$$
\begin{aligned}
P_{m}(x) & =T(x) \bar{P}_{m}(x) \\
= & T(x)\left\{\left[T^{-1}(x) A(x-1) T(x-1)\right]\left[T^{-1}(x-1) A(x-2) T(x-2)\right]\right. \\
& \left.\cdots\left[T^{-1}(x-m+1) A(x-m) T(x-m)\right]\right\} .
\end{aligned}
$$

It is sufficient to prove that the elements of the first column of $\bar{P}_{m}(x)$ will converge, inasmuch as the elements of the first column of $P_{m}(x)$ arise from combination of the rows of $T(x)$ with the first column of $\bar{P}_{m}(x)$. The matrix $\bar{P}_{m}(x)$ is a product of matrices, the type of each of which is

$$
T^{-1}(x+1) A(x) T(x) \text {. }
$$

By (10) and (11) this reduces to

$$
1+\frac{1}{x^{k}} T^{-1}(x) C(x) T(x) .
$$

Let us examine the second term. The element in the $i$ th row and $j$ th column of $T^{-1}(x)$ will be given by an expression like that for $s_{i j}^{\prime}(x)$ in $(9)$ with the saine first $k$ terms; the elements of $C(x)$ are analytic at $x=\infty$, the element 
in the $i$ th row and $j$ th column of $T(x)$ will be given by an expression like that for $s_{i j}(x)$ and with the same first $k$ terms. The element in the $i$ th row and $j$ th column of the product $T^{-1}(x) C(x) T(x)$ has therefore the form

$$
\left(\begin{array}{c}
\rho_{j} \\
\rho_{i}
\end{array}\right)^{x} x^{r,-r_{i}} \text { times a function analytic at } x=\infty \text {. }
$$

Choose first $k$ so large that it exceeds every difference $r_{j}-r_{i}$ in absolute value by more than an assigned integer $d \geqq 2$, and we see that the second term may be written

$$
\frac{1}{x^{d}} \Theta(x) \text { with elements } \theta_{i j}(x)=\left(\frac{\rho_{j}}{\rho_{i}}\right)^{x} \phi_{i j}(x) .
$$

The function $\phi_{i j}(x)$ is finite in the vicinity of $x=\infty$, at least if $x$ be restricted not to pass the positive axis of reals.

By means of this notation, $\bar{P}_{m}(x)$ reduces to the form

$$
\left(1+\frac{1}{(x-1)^{l}} \Theta(x-1)\right)\left(1+\frac{1}{(x-2)^{\prime}} \Theta(x-2)\right) \cdots\left(1+\frac{1}{(x-m)^{l}} \Theta(x-m)\right),
$$

or more fully,

$$
\begin{aligned}
1+\sum_{k_{1}} \frac{1}{\left(x-k_{1}\right)^{l}} & \Theta\left(x-k_{1}\right) \\
& +\sum_{k_{1}, k_{2}}^{k_{1}<k_{2}} \frac{1}{\left(x-k_{1}\right)^{d}\left(x-k_{2}\right)^{d}} \Theta\left(x-k_{1}\right) \Theta\left(x-k_{2}\right)+\cdots
\end{aligned}
$$

It is the elements of the first column of this matrix which must be shown to converge. According to the notation of (15), the $i$ th element is

$$
\begin{aligned}
\delta_{i 1}+\sum_{k_{1}} \frac{1}{\left(x-k_{1}\right)^{\prime \prime}} & \left(\begin{array}{c}
\rho_{1} \\
\rho_{i}
\end{array}\right)^{x-k_{1}} \phi_{i 1}\left(x-k_{1}\right)+\sum_{k_{1}, k_{2}}^{k_{1}<k_{2}} \frac{1}{\left(x-k_{1}\right)^{\prime \prime}\left(x-k_{2}\right)^{\prime l}} \\
& \times \sum_{\tau=1}^{n}\left(\frac{\rho_{\tau}}{\rho_{i}}\right)^{x-k_{1}}\left(\frac{\rho_{1}}{\rho_{\tau}}\right)^{x-k_{2}} \phi_{i \tau}\left(x-k_{1}\right) \phi_{\tau 1}\left(x-k_{2}\right)+\cdots
\end{aligned}
$$

But $\phi_{i j}(x)$ is limited in the vicinity of $x=\infty$ and is less than some constant $M$ for $|x|>R_{1}$. Therefore if all the points $x-1, x-2, \ldots$ are exterior to the circle $|x|=R_{1}$, which is true if $x$ lies in $D$ of fig. 1, the typical element in

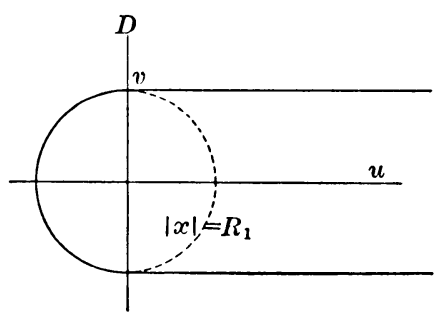

the $(l+1)$ th term of $(17)$,

FI(i. 1 . 


$$
\begin{aligned}
\frac{1}{\left(x-k_{1}\right)^{l}} \frac{1}{\left(x-k_{2}\right)^{l}} \cdots \frac{1}{\left(x-k_{l}\right)^{l}}\left(\frac{\rho_{\tau}}{\rho_{i}}\right)^{x-k_{1}}\left(\frac{\rho_{\sigma}}{\rho_{\tau}}\right)^{x-k_{2}} \cdots\left(\frac{\rho_{1}}{\rho_{\nu}}\right)^{x-k_{l}} \\
\\
\times \phi_{i \tau}\left(x-k_{1}\right) \phi_{\tau \sigma}\left(x-k_{2}\right) \cdots \phi_{\nu 1}\left(x-k_{l}\right),
\end{aligned}
$$

is less in absolute value than the quantity

$$
\left(\frac{\rho_{1}}{\rho_{i}}\right)^{x} \frac{M^{l}}{\left(x-k_{1}\right)^{d} \cdots\left(x-k_{l}\right)^{d}} .
$$

This inequality depends on the fact that we have

$$
\begin{aligned}
& \left|\left(\frac{\rho_{\tau}}{\rho_{i}}\right)^{x-k_{1}}\left(\frac{\rho_{\sigma}}{\rho_{\tau}}\right)^{x-k_{2}} \ldots\left(\frac{\rho_{1}}{\rho_{\nu}}\right)^{x-k_{l}}\right| \\
& \quad=\left|\left(\frac{\rho_{1}}{\rho_{i}}\right)^{x}\left(\frac{\rho_{i}}{\rho_{1}}\right)^{k_{1}}\left(\frac{\rho_{\tau}}{\rho_{1}}\right)^{k_{2}-k_{1}} \cdots\left(\frac{\rho_{\nu}}{\rho_{1}}\right)^{k_{l}-k_{l-1}}\right| \leqq\left|\left(\frac{\rho_{1}}{\rho_{i}}\right)^{x}\right|
\end{aligned}
$$

as a consequence of $(8)$, and the fact that $k_{1}, k_{2}, \ldots, k_{n}$ are an increasing set of positive integers. Hence each term of (17) does not exceed the corresponding term of

$$
\delta_{i 1}+\left|\left(\frac{\rho_{1}}{\rho_{i}}\right)^{x}\right| \sum_{k_{1}} \frac{M}{\left|x-k_{1}\right|^{d}}+\left|\left(\frac{\rho_{1}}{\rho_{i}}\right)^{x}\right| \sum_{k_{1}, k_{2}}^{k_{1}<k_{2}} \frac{n M^{2}}{\left|x-k_{1}\right|^{d}\left|x-k_{2}\right|^{d}}+\cdots
$$

in absolute value.

Allow $m$ to approach infinity. The expression (17) becomes a multiple series. Since the sum of the terms after the first in (18) approaches the limit

$$
\frac{1}{n}\left|\left(\frac{\rho_{1}}{\rho_{i}}\right)^{x}\right|\left\{\left[\left(1+\frac{n M}{|x-1|^{d}}\right)\left(1+\frac{n M}{|x-2|^{d}}\right) \cdots\right]-1\right\},
$$

it is obvious that the elements of the first column of $\bar{P}_{m}(x)$ and therefore of $P_{m}(x)$ converge absolutely and uniformly to an analytic function in the vicinity of points in $D$. Moreover as we may write

$$
P_{m}(x)=A(x-1) A(x-2) \cdots A(x-r) P_{m-r}(x-r),
$$

and as we may take $r$ so large that $x-r-1, x-r-2, \ldots$ lie without the circle $|x|=R_{1}$, the elements of the first column of $P_{m}(x)$ will converge uniformly in the vicinity of any point of the plane excepting those only at which one of the elements of $A(x-1), A(x-2), \ldots$ has a pole. Hence the $i$ th element of the first column of $P_{m}(x)$ converges to a function $u_{i}(x)$ which is analytic except at the points congruent to (any number of units to right or left of) the poles of the elements of $A(x)$ on the right. At these points $u_{i}(x)$ may have a pole.

It may easily be made evident that the definition of $u_{1}(x), u_{2}(x), \ldots, u_{n}(x)$ 
is independent of the choice of $k$ and $T$. For if $k$ is any second choice of $k$ and $T^{\prime}(x)$ is the corresponding function $T(x)$, we may nevertheless write

$$
\begin{aligned}
& P^{\prime \prime \prime}(x)=T(x)\left\{\left[T^{-1}(x) A(x-1) T(x-1)\right]\right. \\
& \quad \times\left[T^{-1}(x-1) A(x-2) T(x-2)\right] \cdots\left[T^{-1}(x-m+1) A(x-m) T^{\prime}(x-m)\right] .
\end{aligned}
$$

If the elements of the first column be expanded in a sum like (17) and $m$ be allowed to become infinite, the resulting multiple series for $u_{i}(x)$ will be term for term identical with that obtained before.

It remains to discuss the asymptotic nature of $u_{1}(x), \ldots, u_{n}(x)$ for large values of $|x|$. The limit $\bar{u}_{i}(x)$ of the $i$ th element of the first column of $\bar{P}_{m}(x)$ differs from $\delta_{i 1}$ by a quantity less than (19) in absolute value if $x$ lies in $D$. This quantity is itself less than

$$
\frac{1}{n}\left(\frac{\rho_{1}}{\rho_{i}}\right)^{x}\left(e^{\sum_{\nu=0}^{\infty} \frac{n M}{|x-\nu|^{d}}}-1\right)
$$

in absolute value. If now any line be drawn parallel to the axis of imaginaries and if $x$ be restricted to lie in $D$ to the left of the line, by inequality (12) the last factor will be of the form $M(x) / x^{d-1}$, where $M(x)$ is limited. Thus we may write

$$
\bar{u}_{i}(x)=\delta_{i 1}+\left(\frac{\rho_{1}}{\rho_{i}}\right)^{x} \frac{M_{i}(x)}{x^{d-1}}, \quad\left|M_{i}(x)\right|<M
$$

for $x$ in $D$ and to the left of this line. Likewise if $x$ is in $D$ to the right of such a line, by inequality (13) we may write

$$
\bar{u}_{i}(x)=\delta_{i 1}+\left(\frac{\rho_{1}}{\rho_{i}}\right)^{x} \frac{M_{i}^{\prime}(x)}{v^{\prime-1}}, \quad\left|M_{i}^{\prime}(x)\right|<M .
$$

But the relation $P_{m}(x)=T(x) \bar{P}_{m}(x)$ gives at once

$$
u_{i}(x)=\sum_{\tau=1}^{n} t_{i \tau}(x) \bar{u}_{\tau}(x) .
$$

If in this equation the first of the above expressions for $\bar{u}_{1}(x), \bar{u}_{2}(x), \ldots, \bar{u}_{n}(x)$ be substituted, we find at once

$$
u_{i}(x)=t_{i 1}(x)+\sum_{\tau=1}^{n} t_{i \tau}(x)\left(\frac{\rho_{1}}{\rho_{\tau}}\right)^{x} \frac{M_{\tau}(x)}{x^{d-1}} .
$$

The functions $t_{i j}(x)$ are the same in form as $8_{i j}(x)$ up to the $k$ th term. Substituting in their expansions we obtain

$$
u_{i}(x)=x^{\mu x} \rho_{1}^{r} x^{r_{1}}\left\{\left(s_{i 1}+\frac{s_{i 1}^{(1)}}{x}+\cdots\right)+\sum_{\tau=1}^{n} \frac{x^{r_{\tau}}}{x^{r_{1}}} \frac{M_{\tau}(x)}{x^{(l-1}}\left(s_{i \tau}+\frac{s_{i \tau}^{(1)}}{x}+\cdots\right)\right\} .
$$

The second term in brackets can clearly be made infinitesimal in $x$ of as high 
order as may be desired by taking $k$, and therefore $d$, large enough. Consequently $u_{i}(x)$ is represented asymptotically by $s_{i 1}(x)$ with respect to $x$ to the left of any such line and likewise with respect to $v$ to the right of such a line. The part of the theorem that relates to the case $\lambda=1$ is proved.

It is clear that if the subscripts $1,2, \ldots, n$ had not been so chosen that (8) was true, the only modification would be that the elements of any $i$ th column of $P_{m}(x)$ for which the quantity $\rho_{i}$ had a maximum absolute value would converge.

The case $\lambda=2$ will next be discussed. This reduces at once to the case $\lambda=1$ inasmuch as the two-rowed determinants $u_{a \beta}^{(m)}(x)$ formed from the elements of the first two columns of $P^{(m)}(x)$ which are in the ath and $\beta$ th rows $(\alpha<\beta)$ are related to a certain difference system precisely as the elements of the first column are related to the original system (1).

In fact, form the system with general solution

$$
g_{i j}(x)=\left|\begin{array}{ll}
g_{i}^{\prime}(x) & g_{i}^{\prime \prime}(x) \\
g_{j}^{\prime}(x) & g_{j}^{\prime \prime}(x)
\end{array}\right| \quad(i, j=1,2, \cdots, n ; i<j) .
$$

Here $g_{1}^{\prime}(x), g_{2}^{\prime}(x), \cdots, g_{n}^{\prime}(x)$ and $g_{1}^{\prime \prime}(x), g_{2}^{\prime \prime}(x), \cdots, g_{n}^{\prime \prime}(x)$ are any pair of solutions of (1). One finds by the use of (1)

where

$$
g_{i j:}(x+1)=\sum_{k, l} a_{i j: k l}(x) g_{k l:}(x)
$$

$$
a_{i j: k l}(x)=a_{i k}(x) a_{j l}(x)-a_{i l}(x) a_{j k}(x) .
$$

Accordingly we have $\frac{1}{2} n(n-1)$ linear difference equations of precisely the type (1) to determine the functions $g_{i j}:(x)$. It is clear that the sets of formal expansions

$$
\begin{aligned}
s_{i j: k l}(x) & =\left|\begin{array}{ll}
s_{i k}(x) & s_{i l}(x) \\
s_{j k}(x) & s_{j l}(x)
\end{array}\right| \\
& =x^{2 \mu x}\left(\rho_{k} \rho_{l}\right)^{x} x^{r_{k}+l l}\left\{\left(s_{i k} s_{j l}-s_{i l} s_{j k}\right)+\text { terms in } x^{-1}, x^{-2}, \ldots\right\},
\end{aligned}
$$

$k$ and $l$ fixed, will satisfy (21). There are $\frac{1}{2} n(n-1)$ sets of these, and furthermore the determinant of the constant terms

$$
\left|s_{i k} s_{j l}-s_{i l} s_{i k}\right|
$$

is precisely equal to $d^{n-1}$ and is therefore not zero by (7).* Hence the system (21) satisfies the restrictive hypothesis made at the beginning of $\S 1$.

We are now in a position to apply the result alieady obtained. In matrix notation, equations (21) may be expressed as

$$
G_{2}(x+1)=A_{2}(x) G_{2}(x) \text {. }
$$

* Pascal : Die Veterminanten, p. 87. 
Also let $T_{2}(x)$ be the matrix which the formal matrix solution $S_{2}(x)$ becomes when the formal series are replaced by convergent series which agree with them to the $k$ th term. The elements in that column of

$$
P_{2 m}(x)=A_{2}(x-1) A_{2}(x-2) \cdots A_{2}(x-m) T_{2}(x-m)
$$

for which the quantity $\rho_{k} \rho_{l}$ in the formal solution which appears in the same column of $S_{2}(x)$ has a maximum absolute value will converge; this results from an application of the preceding result for $\lambda=1$ to the system of $\frac{1}{2} n(n-1)$ equations. Equations (8) show that we may take $k=1, l=2$ and it is also clear that we may take the elements of this column of $T_{2}(x)$ to be

$$
t_{i j: 12}(x)=\left|\begin{array}{ll}
t_{i 1}(x) & t_{i 2}(x) \\
t_{j 1}(x) & t_{j 2}(x)
\end{array}\right| .
$$

The elements $p_{i j: 12}^{(m)}(x)$ of this column of $P_{2 m}(x)$ converge to functions analytic everywhere except for poles at points to the right of the poles of the elements of $A_{2}(x)$ and congruent to them. Moreover these functions are represented asymptotically by $s_{i j: 12}(x)$ throughout the entire plane, with respect to $x$ to the left of any line parallel to the axis of imaginaries, and with respect to $v$ to the right of such a line.

But the sequence $p_{i j: 12}^{(m)}(x), m=1,2, \ldots$ is identical with the sequence of determinants $u_{i j}^{(m)}(x)$ formed from the elements of the first two columns which are in the $i$ th and $j$ th rows of $P_{m}(x)$. In fact the first member of both sequences is $t_{i j: 12}(x)$, and in general $p_{i j: 12}^{(m+1)}(x)$ is derived from $p_{i j: 12}^{(n)}(x)$ just as $u_{i j}^{(m+1)}(x)$ is derived from $u_{i j}^{(m)}(x)$, namely we have

$$
\begin{aligned}
& p_{i j: 12}^{(m+1)}(x)=\sum_{l i, l} a_{i j: k l}(x-1) p_{k l: 12}^{(m)}(x-1), \\
& u_{i j}^{(m+1)}(x)=\left|\begin{array}{l}
p_{i 1}^{(m+1)}(x), p_{i 2}^{(m+1)}(x) \mid \sum_{\tau=1}^{n} a_{i \tau}(x-1) p_{\tau 1}^{(m)}(x-1), \sum_{\tau=1}^{n} a_{i \tau}(x-1) p_{\tau 2}^{(m)}(x-1) \\
p_{j 1}^{(m+1)}(x), p_{j 2}^{(m+1)}(x)
\end{array}\right|=\begin{array}{l}
\sum_{\tau=1}^{n} a_{j \tau}(x-1) p_{\tau 1}^{(m)}(x-1), \sum_{\tau=1}^{n} a_{j \tau}(x-1) p_{\tau 2}^{(m)}(x-1)
\end{array} \mid \\
& =\sum_{l i, l} a_{i j: k l}(x-1) u_{k l l}^{(m)}(x-1) .
\end{aligned}
$$

Thus the theorem is true for $\lambda=2$ also. Strictly speaking, we have only obtained functions $u_{i j}(x)$ for $i<j$; but we have obviously such functions for all $i$ and $j$; these may be obtained by means of the equation $u_{i j}(x)=-u_{j i}(x)$. In like manner the theorem can be demonstrated for the remaining values of $\lambda$.

The existence of the functions $v_{i j \ldots l}(x)$ and their properties may be eitablished in the same way. The rolle of right and left in the $x$-plane will be interchanged and the equation (4)' replaces (4). 
The functions $u_{i j \ldots l}(x)$ and $v_{i j \ldots l}(x)$ will be called the determinant limits on account of their origin.

In the case that the restrictive hypothesis is satisfied and all the roots $\rho$ of the characteristic equation are equal, Theorem I supplies a complete existence theorem, every column of elements of $P_{m}(x)$ and $Q_{m}(x)$ converging to an analytic solution of (1). These conditions are met if one has

$$
a_{i j}(x)=\delta_{i j}+\frac{a_{i j}^{(2)}}{x^{2}}+\frac{a_{i j}^{(3)}}{x^{3}}+\cdots
$$

In general however Theorem I supplies only one solution directly, and that is furnished by

$$
g_{1}(x)=u_{1}(x), g_{2}(x)=u_{2}(x), \cdots g_{n}(x)=u_{n}(x) .
$$

This set of functions must be a solution since the definition of $u_{i}(x)$ gives us

$$
\begin{aligned}
u_{i}(x+1) & =\lim _{m=\infty} \sum_{\tau, \ldots, \eta=1}^{n} a_{i \tau}(x) \cdots a_{\nu \eta}(x-m) t_{\eta 1}(x-m) \\
& =\sum_{\tau=1}^{n} a_{i \tau}(x) u_{\tau}(x) .
\end{aligned}
$$

\section{§2. The Solutions Associated with the Determinant Limits.}

The functions $u_{i}(x), u_{i j}(x), \ldots$ which have been obtained behave as though they were constituent determinants of a matrix of solutions of (2).

Theonem II. There exist $n$ solutions $g_{1 j}(x), g_{2 j}(x), \cdots, g_{n j}(x)\left[h_{1 j}(x)\right.$, $\left.h_{2 j}(x), \cdots, h_{n j}(x)\right](j=1,2, \cdots, n)$ of $(1)$ over any discrete set of values congruent to a given value $x_{0}$ of $x$, such that every $\lambda$-rowed determinant formed from the first [last] $\lambda$-columns of the matrix $G(x)[H(x)]$ and the ith, jth, $\ldots$, lth rows will equal $u_{i j \ldots l}(x)\left[v_{i j \ldots l}(x)\right]$ at these points. The first solution $g_{11}(x), g_{21}(x), \cdots, g_{n 1}(x)\left[h_{1 n}(x), h_{2 n}(x), \cdots, h_{n n}(x)\right]$ is uniquely determined, the second solution $g_{12}(x), g_{22}(x), \cdots, g_{n 2}(x)\left[h_{1, n-1}(x)\right.$, $\left.h_{2, n-1}(x), \ldots, h_{n, n-1}(x)\right]$ is determined up to an additive term obtained by multiplying all the corresponding elements of the first solution by an arbitrary finction of period 1, and so on, any one of these solutions being determined up to an additive term linear in the corresponding elements of the preceding solutions with arbitrary multipliers of period 1.

Proof. The theorem is true in so far as it relates to $\lambda=1$ since $u_{1}(x)$, $u_{2}(x), \cdots, u_{n}(x)$ has been seen to give a solution of $(1)$. We may write then

$$
g_{11}(x)=u_{1}(x), g_{21}(x)=u_{2}(x), \cdots, g_{n 1}(x)=u_{n}(x) \text {. }
$$

Next we shall demonstrate that there exists some solution $g_{12}(x), g_{22}(x), \ldots$, $g_{n 2}(x)$ such that for all $i$ and $j$ 


$$
\left|\begin{array}{ll}
g_{i 1}(x) & g_{i 2}(x) \\
g_{j 1}(x) & g_{j 2}(x)
\end{array}\right|=u_{i j}(x) \text {. }
$$

For this purpose we shall recur to the sequence of elements of $P_{m}(x)$. By means of the identity

$$
\left|\begin{array}{lll}
p_{i 1}^{(m)}(x) & p_{i 2}^{(m)}(x) & p_{i 1}^{(m)}(x) \\
p_{j 1}^{(m)}(x) & p_{j 2}^{(m)}(x) & p_{j 1}^{(m)}(x) \\
p_{k 1}^{(m)}(x) & p_{k: 2}^{(m)}(x) & p_{k i 1}^{(m)}(x)
\end{array}\right|=0
$$

we infer, by developing according to the elements of the third column and letting $m$ become infinite, that

$$
u_{i j}(x) u_{k}(x)-u_{i k}(x) u_{j}(x)+u_{j k}(x) u_{i}(x)=0 \quad(i, j, k=1,2, \cdots, n) .
$$

The $n$ quantities $g_{12}\left(x_{0}\right), g_{22}\left(x_{0}\right), \ldots, g_{n 2}\left(x_{0}\right)$ uniquely determine a solution of (1) on the set of points congruent to $x_{0}$. Choose these $n$ quantities so that (22) is satisfied for some fixed $i$ such that $g_{i 1}\left(x_{0}\right) \neq 0$, and for every $j$. All the rest of the equations (22) will then be satisfied, as is evident from the preceding equation. This gives us

$$
\left|\begin{array}{ll}
g_{i 1}(x) & g_{i 2}(x) \\
g_{j 1}(x) & g_{j 2}(x)
\end{array}\right| g_{k 1}(x)-\left|\begin{array}{ll}
g_{i 1}(x) & g_{i 2}(x) \\
g_{k 1}(x) & g_{k 2}(x)
\end{array}\right| g_{j 1}(x)+u_{j k}(x) g_{i 1}(x)=0
$$

for $x=x_{0}$, which reduces to

$$
u_{j k}(x)=\left|\begin{array}{ll}
g_{j 1}(x) & g_{j 2}(x) \\
g_{k 1}(x) & g_{k 2}(x)
\end{array}\right|
$$

for $x=x_{0}$. Hence $g_{i j}(x)$ and $u_{i j}(x)$ are equal at $x=x_{0}$ for all $i$ and $j$. But they are both constituent elements of a solution of (21), and therefore will agree at all congruent points, since only one solution of that equation exists on the discrete set of points congruent to $x_{0}$ whose elements have given values at this point. It is necessary to make an exception of points congruent to the poles of $A(x)$ or $A^{-1}(x-1)$, and such points are excluded.

The method by which this second solution was obtained clearly allows us to add to each element $g_{i 2}(x)$ a term of the form $p(x) g_{i 1}(x)$, where $p(x)$ is of period 1.

One may now in an entirely analogous way define a solution $g_{13}(x), g_{23}(x)$, $\cdots, g_{n 3}(x)$ on the set of points congruent to $x_{0}$. The identities to be employed in this case are

$$
u_{i j k}(x) u_{l}(x)-u_{i j l}(x) u_{k}(x)+u_{i k l}(x) u_{j}(x)-u_{j k l}(x) u_{i}(x)=0 .
$$

The function $g_{i 3}(x)$ is only determined up to an additive term

$$
p(x) g_{i 1}(x)+q(x) g_{i 2}(x) \text {. }
$$


By proceeding thus the theorem may be proved in a succession of steps in so far as it relates to the elements $g_{i j}(x)$.

The part of the theorem that relates to the elements $h_{i j}(x)$ is proved in an entirely similar way.

The solutions $g_{1 j}(x), \cdots, g_{n j}(x)\left[h_{1 j}(x), \cdots, h_{n j}(x)\right]$ may be said to be the solutions associated with the determinant limits $u_{i}(x), u_{i j}(x) \cdots\left[v_{i}(x)\right.$, $\left.v_{i j}(x) \cdots\right]$.

It is important to obtain explicit formulas for these functions $g_{i j}(x)$, and we shall show that it is possible to obtain the solutions $g_{1 j}(x), g_{2 j}(x), \ldots$, $g_{n j}(x)$ for $j=1,2, \ldots, n$ in terms of $u_{i}(x), u_{i j}(x), \cdots, u_{12 \ldots n}$, and the operation $\sum$ defined by the condition

$$
\sum_{t=x+1} \phi(t)-\sum_{t=x} \phi(t)=\phi(x) .
$$

The functions $g_{11}(x), \cdots, g_{n 1}(x)$ are equal respectively to $u_{1}(x), \ldots, u_{n}(x)$. It will accordingly be sufficient to obtain formulas for $g_{1 k}(x), \cdots, g_{n k}(x)$ in terms of the solutions $g_{1 j}(x), \cdots, g_{n j}(x)(j=1,2, \cdots, k-1)$ and the determinant limits.

Form the determinant

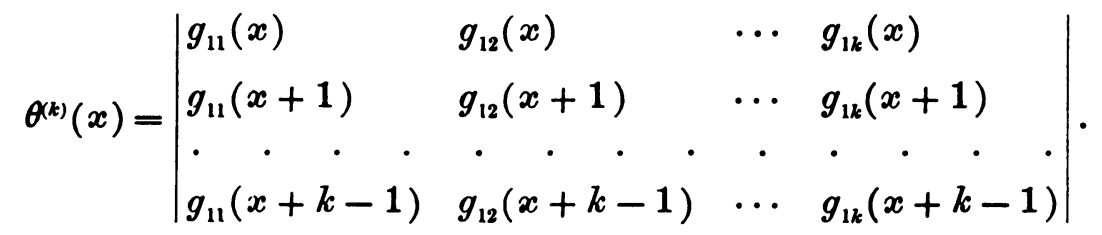

If now in place of the elements $g_{i j}(x+m)(m>0)$ we insert their value as obtained from (1)

$$
g_{1 j}(x+m)=\sum_{\tau, \sigma, \ldots, \theta=1}^{n} a_{1 \tau}(x+m-1) a_{\tau \sigma}(x+m-2) \cdots a_{\eta \theta}(x) g_{\theta j}(x),
$$

in terms of the functions $g_{1 j}(x), \cdots, g_{n j}(x)$, we see that the above determinant may be expressed as a sum of terms each of which contains as factor a determinant which reduces to one of the determinant limits of order $k$. In fact we have obviously

$$
\theta^{(k)}(x)=\sum_{\tau, \sigma, \ldots=1}^{n}\left\{a_{1 \tau}(x)\left[\sum_{\tau=1}^{n} a_{1 \tau}(x+1) a_{\tau \sigma}(x)\right] \ldots\right\} u_{\tau \sigma} \ldots(x) .
$$

Hence this determinant is to be regarded as a known function.

But the identity (23) may also be looked upon as a non-homogeneous linear difference equation of order $k-1$ in $g_{1 k}(x)$, and may be written

$$
\theta^{(k-1)}(x) g_{1 k}(x+k-1)+\psi(x) g_{1 k}(x+k-2)+\cdots \pm \theta^{(k-1)}(x+1) g_{1 k}(x)=\theta^{(k)}(x) \text {. }
$$

The associated reduced homogeneous equation obtained by equating the left 
hand member to zero has the solutions [see (23)] $g_{11}(x), \cdots, g_{1, k-1}(x)$, which are linearly independent unless $\theta^{(k-1)}(x)$ is zero. By a method analogous to that of variation of parameters for ordinary linear differential equations, let us put

$$
g_{1 k}(x)=\sum_{j=1}^{k-1} g_{1 j}(x) l_{j}(x)
$$

and determine $l_{1}(x), l_{2}(x), \cdots, l_{k-1}(x)$ by the equations

$$
\begin{gathered}
\sum_{j=1}^{k-1} g_{1 j}(x+1) \Delta l_{j}(x)=0, \\
\sum_{j=1}^{k-1} g_{1 j}(x+2) \Delta l_{j}(x)=0, \\
\cdot \quad \cdot \quad \cdot \quad \cdot \quad \cdot \quad \cdot \\
\sum_{j=1}^{k-1} g_{1 j}(x+k-1) \Delta l_{j}(x)=\begin{array}{c}
\theta^{(k)}(x) \\
\theta^{(k-1)}(x)
\end{array},
\end{gathered}
$$

where as usual $\Delta \phi$ denotes the difference $\phi(x+1)-\phi(x)$. There are here $k-1$ linear equations to determine the $k-1$ unknowns $\Delta l_{1}(x), \ldots, \Delta l_{k-1}(x)$, and their determinant is precisely $\theta^{(k-1)}(x+1)$. Unless this determinant is identically zero, we have accordingly

$$
\Delta l_{j}(x)=\frac{\theta^{(k)}(x) m_{j, k-1}(x)}{\theta^{(k-1)}(x) \theta^{(k-1)}(x+1)},
$$

in which $m_{j, k-1}(x)$ is the cofactor of the element in the $j$ th column and last row of the determinant $\theta^{(k-1)}(x+1)$ :

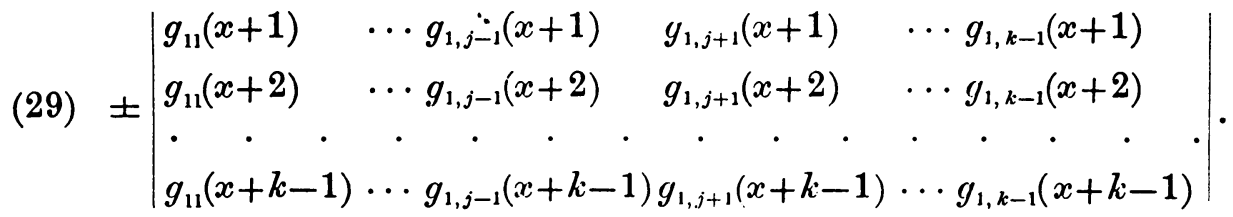

The resulting expression for $g_{1 k}(x)$ is

$$
g_{1 k}(x)=\sum_{j=1}^{k-1} g_{1 j}(x) \sum_{t=x} \frac{\theta^{(k)}(t) m_{j, k-1}(t)}{\theta^{(k-1)}(t) \theta^{(k-1)}(t+1)}
$$

It remains to show that $g_{1 k}(x)$ as thus defined is a solution of (26). We have

$$
\begin{aligned}
g_{1 k}(x) & =\sum_{j=1}^{k-1} g_{1 j}(x) l_{j}(x), \\
g_{1 k}(x+1) & =\sum_{j=1}^{k-1}\left[\left(g_{1 j}(x+1) l_{j}(x)+g_{1 j}(x+1) \Delta l_{j}(x)\right]\right. \\
& =1 \sum_{j=1}^{k-1} g_{1 j}(x+1) l_{j}(x),
\end{aligned}
$$




$$
\begin{aligned}
g_{1 k}(x+k-1)=\sum_{j=1}^{k-1}\left[\left(g_{1 j}(x+k-1)\right.\right. & \left.l_{j}(x)+g_{1 j}(x+k-1) \Delta l_{j}(x)\right] \\
& =\sum_{j=1}^{k_{k-1}} g_{i j}(x+k-1) l_{j}(x)+\frac{\theta^{(k)}(x)}{\theta^{(k-1)}(x)} .
\end{aligned}
$$

Hence, if we multiply the last of these equations by $\theta^{(k-1)}(x)$, the one which precedes it by $\psi(x)$ and so on, and add corresponding members, the left hand member of the resulting equation will be precisely the left hand member of (26). Also all the terms except the last of the right hand member reduce to zero by reason of the fact that $g_{11}(x), \cdots, g_{1, k-1}(x)$ are solutions of the reduced equation, and there remains only a term $\theta^{(k)}(x)$. Therefore $g_{1 k}(x)$ is the desired solution : furthermore the formula $(30)$ does not determine $g_{1 k}(x)$ uniquely but only up to a sum of terms

$$
p_{1}(x) g_{11}(x)+\cdots+p_{k-1}(x) g_{1, k-1}(x),
$$

where $p_{1}(x), \cdots, p_{k-1}(x)$ are arbitrary periodic multipliers since the operation $\sum_{t=x}$ is determined only up to an additive periodic term. But the result stated in Theorem II shows that $g_{1 k i}(x)$ has precisely this degree of indeterminateness, and hence the most general determination of $g_{1 k}(x)$ is furnished by (30) unless $\theta^{(k-1)}(x)$ is identically zero, and this is an obvious case of failure.

When a choice of $g_{1 k}(x)$ has been made, the remaining functions $g_{2 k}(x), \ldots$, $g_{n k}(x)$ which make up the $k$ th solution are determined. In fact the equations (24) for $m=1,2, \cdots, n$ form $n$ linear equations in $g_{1}(x), \cdots, g_{n}(x)$ of determinant not zero, since in the contrary case we should obtain a linear homogeneous relation between $g_{1}(x+1), \cdots, g_{1}(x+n)$, which contradicts the condition placed on $g_{1}(x)$ at the outset that it satisfies an equation of the $n$th order. By solving these equations we obtain

$$
\begin{array}{r}
g_{i}(x)=\sigma_{i 1}(x) g_{1}(x+1)+\sigma_{i 2}(x) g_{1}(x+2)+\cdots+\sigma_{i n}(x) g_{1}(x+n) \\
(i=1,2, \cdots, n),
\end{array}
$$

where the functions $\sigma_{i j}(x)$ are rational in $x$. These equations determine $g_{2}(x), \cdots, g_{n}(x)$ in terms of $g_{1}(x)$.

That none of functions $\theta^{(1)}(x), \ldots, \theta^{(n)}(x)$ can be identically zero may be proved as follows: In the first place $\theta^{\left(k_{i}\right)}(x)$ has a definite asymptotic form in the entire $x$-plane, namely that obtained by replacing $u_{\tau \sigma} \ldots(x)$ by $s_{\tau \sigma} \ldots(x)$ in the right hand member of (25). This is a consequence of Theorem I. But this form is

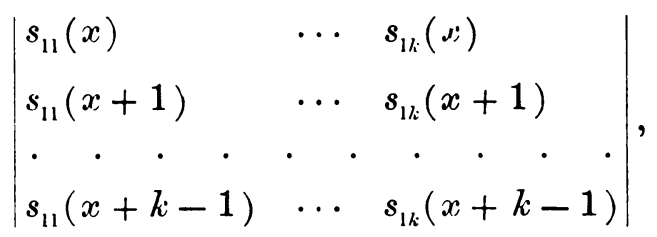


as may be shown readily by means of (23). Recalling now the explicit form of the series $s_{i j}(x)$, we obtain

$$
\theta^{(k)}(x) \sim x^{k_{\mu} x}\left(\rho_{1} e^{-\mu}\right)^{x} \cdots\left(\rho_{k} e^{-\mu}\right)^{x} x^{r_{1}+\ldots+r_{k}}\left(d_{k}+\frac{d_{k}^{(1)}}{x}+\cdots\right) .
$$

Since $\theta^{(k)}(x)$ is represented asymptotically by this expression with respect to $x$ to the left of any line parallel to the axis of imaginaries, and with respect to $v$ to the right of such a line (Theorem I), it is obvious that unless $d_{k}, d_{k}^{(1)}, \ldots$ are all zero, $\theta^{(k)}(x)$ does not vanish at a great distance from the positive axis of reals. But these constants can not all vanish. Otherwise the above determinant vanishes (formally) and $s_{11}(x), \cdots, s_{1 k}(x)$ are linearly dependent, and accordingly, by $(32), s_{i 1}(x), \ldots, s_{i k}(x)(i=1,2, \ldots, n)$, are linearly dependent, in contradiction to ( 7$)$. Thus (30) is a valid formula.

\section{§3. The Intermediate Associated Solutions.}

The application of the formulas (30) and (32) leads us immediately to a set of intermediate associated solutions

$$
g_{1 k}^{\prime}(x), \cdots, g_{n k}^{\prime}(x) \quad(k=1,2, \cdots, n),
$$

analytic above and below lines $A \infty$ and $B \infty$ parallel to the axis of reals, and which have the property that

$$
g_{1 k}^{\prime}(x) \sim s_{1 k}(x), \cdots, g_{n k}^{\prime}(x) \sim s_{n k}(x) \quad(k=1,2, \cdots, n),
$$

with respect to $x$ in any left half plane and with respect to $v$ in any right half plane.

We take

$$
g_{11}^{\prime}(x)=u_{1}(x), \cdots, g_{n 1}^{\prime}(x)=u_{n}(x),
$$

and this first solution has the properties stated, by Theorem 1 . We now define the solutions $g_{12}^{\prime}(x), \ldots, g_{n 2}^{\prime}(x) ; g_{13}^{\prime}(x), \ldots, g_{n 3}^{\prime}(x) ; \ldots$ in succession by choosing the summation operation $\Sigma$ on $\phi(x)$ to be defined as

$$
-\phi(x)-\phi(x+1)-\cdots
$$

and employing formulas (30) and (32). It will suffice to show that a solution $g_{1 j}^{\prime}(x), \cdots, g_{n j}^{\prime}(x)$ for $j=k$ will exist and have these properties under the hypothesis that the same is true for $j=1,2, \ldots, k-1$. The formula (30), which serves to define $g_{1 k}^{\prime}(x)$ if $\left|\rho_{k-1}\right|>\left|\rho_{k}\right|$, is then

$$
g_{1 k}^{\prime}(x)=-\sum_{j=1}^{k-1} g_{1 j}^{\prime}(x) \sum_{\nu=0}^{\infty} \frac{\theta^{(k)}(x+\nu) m_{j, k-1}^{\prime}(x+\nu)}{\theta^{(k-1)}(x+\nu) \theta^{(k-1)}(x+\nu+1)} .
$$

We have written $m_{j, k-1}^{\prime}(x)$ to stand for what $m_{j, k-1}(x)$ becomes when $g_{i j}^{\prime}$ replaces $g_{i j}$. We shall first prove that the $k-1$ series which appear here are 
convergent. To this end we need to compute the asymptotic form of the typica' term of the above series

$$
\frac{\theta^{(k)}(x) m_{j, k-1}^{\prime}(x)}{\theta^{(k-1)}(x) \theta^{(k-1)}(x+1)},
$$

and this is done by merely substituting the known asymptotic forms for the $\theta$ 's as given in (33) and at the same time the known form of the elements $m_{j, k-1}^{\prime}$. This latter [see (29)] is clearly given by

$$
\begin{aligned}
m_{j, k-1}^{\prime}(x) \sim x^{(k-2) \mu x}\left(\rho_{1} e^{-\mu}\right)^{x} \cdots\left(\rho_{j-1} e^{-\mu}\right)^{x}\left(\rho_{j+1} e^{-\mu}\right)^{x} \\
\cdots\left(\rho_{k-1} e^{-\mu}\right)^{x} x^{r_{1}+\ldots+r_{j-1}+r_{j+1}+\ldots+r_{k-1}}\left\{m_{j}+\frac{m_{j}^{(1)}}{x}+\cdots\right\}
\end{aligned}
$$

as a result of our hypothesis. Combining (35) and (33) we find for the above term the asymptotic formula

$$
\frac{\theta^{(k)}(x) m_{j, k-1}^{\prime}(x)}{\theta^{(k-1)}(x) \theta^{(k-1)}(x+1)} \sim\left(\frac{\rho_{k}}{\rho_{j}}\right)^{x} x^{r_{k}-r_{j}} x^{s_{j}}\left\{\eta_{j}+\frac{\eta_{j}^{(1)}}{x}+\cdots\right\} .
$$

The integer $s_{j}$ is zero unless $d_{k-1}=0$.

Since we have $\left|\rho_{j}\right|>\left|\rho_{k}\right|$ for $j<k$ except when $\left|\rho_{k-1}\right|=\left|\rho_{k}\right|$ [see (8)], the terms in (34) clearly diminish in approximately geometrical ratio for $v$ large enough, and in consequence the series converge uniformly in the vicinity of such points. Thus $g_{1 k}^{\prime}(x)$ has been actually defined and is analytic above and below suitable lines $A \infty$ and $B \infty$.

By the aid of relations (36) we may now determine the asymptotic form of each of the series in (34). In the first place, if each term of the $j$ th series is replaced by its asymptotic formula, the series becomes

$$
\sum_{\nu=0}^{\infty}\left(\frac{\rho_{k}}{\rho_{j}}\right)^{x+\nu}(x+\nu)^{r_{k}-r_{j}}(x+\nu)^{s_{j}}\left(\eta_{j}+\frac{\eta_{j}^{(1)}}{x+\nu}+\cdots\right) .
$$

The error committed in the final bracketed series by breaking off at the $l$ th term is of order $(x+\nu)^{-l}$ if $x$ lies to the left of the axis of imaginaries, and of order $v^{l}$ if $x$ lies to the right of this line, since the imaginary coefficient $v$ of $x+\nu$ is the same as the imaginary coefficient of $x$. These bracketed series are, of course, finite above and below suitable lines $A \infty$ and $B \infty$.

Now separate the series into two parts : first the sum of the first $m$ terms in which $\nu<\frac{1}{2}|x|$, secondly, the remaining terms.

In this first set of terms we have

$$
(x+\nu)^{\rho}=x^{\rho}\left(1+\frac{\rho \nu}{x}+\cdots\right),
$$

where the error committed by breaking off the series at the $k$ th term is of the $\operatorname{order}(\nu / x)^{k}$. Hence the first $m$ terms of the above series may be written 


$$
\left(\frac{\rho_{k}}{\rho_{j}}\right)^{x} x^{r_{k}-r_{j}} x^{s_{j}} \sum_{\nu=0}^{m}\left(\frac{\rho_{k}}{\rho_{j}}\right)^{\nu}\left[\psi_{j}+\frac{\psi_{j}^{(1)}}{x}+\cdots+M_{j}(x)\left(\frac{\nu}{x}\right)^{k}\right],
$$

where $M_{j}(x)$ is limited. Now the terms involving $M_{j}(x)$ are of the order $x^{-k}$, since

$$
\left|\sum_{\nu=0}^{m}\left(\frac{\rho_{k}}{\rho_{j}}\right)^{\nu} M_{j}(x)\left(\frac{\nu}{x}\right)^{k}\right| \leqq \frac{M}{x^{k}} \sum_{\nu=0}^{\infty}\left(\frac{\rho_{k}}{\rho_{j}}\right)^{\nu} \nu^{k} \mid, M \text { a fixed constant. }
$$

As $m$ becomes large we have also

$$
\sum_{\nu=0}^{m}\left(\frac{\rho_{k}}{\rho_{j}}\right)^{\nu} \psi \sim \frac{\rho_{j} \psi}{\rho_{j}-\rho_{k}}
$$

0 all powers of $x$ since the difference in absolute value is

$$
\left|\frac{\left(\frac{\rho_{k}}{\rho_{j}}\right)^{m+1} \psi}{1-\frac{\rho_{k}}{\rho_{j}}}\right|<\left|\frac{\left(\frac{\rho_{k}}{\rho_{j}}\right)^{\frac{x}{2}} \psi}{1-\frac{\rho_{k}}{\rho_{j}}}\right| .
$$

Hence the first $m$ terms have the asymptotic form

$$
\left(\frac{\rho_{k}}{\rho_{j}}\right)^{x} x^{r_{k}-r_{j}} x^{s_{j}}\left\{\psi_{j}+\frac{\psi_{j}^{(1)}}{x}+\cdots\right\}
$$

with respect to $x$ for $x$ to the left of the axis of imaginaries, or similarly to the left of any parallel line. The terms after the $m$ th diminish in approximately geometrical ratio for $|v|$ sufficiently large, and all contain a very small multiplier $\left(\rho_{k} / \rho_{j}\right)^{m}$. Hence the second group of terms does not affect the above asymptotic form.

Substituting the above expression in (34) and at the same time the known asymptotic formulas $s_{1 j}(x)$ for $g_{1 j}^{\prime}(x)$, we find

$$
g_{1 k}^{\prime}(x) \sim \sum_{j=1}^{k-1} x^{\mu . x}\left(\rho_{j} e^{-\mu}\right)^{x} x^{r_{j}}\left\{s_{1 j}+{ }_{a !}^{s_{1, j}^{(1)}}+\cdots\right\}
$$

or multiplying out,

$$
\times\left(\frac{\rho_{k}}{\rho_{j}}\right)^{x} x^{r_{k}-r_{j}} x^{s_{j}}\left\{\psi_{j}+\frac{\psi_{j}^{(1)}}{x}+\cdots\right\},
$$

$$
g_{1 k}^{\prime}(x) \sim\left(\rho_{k} e^{-\mu}\right)^{x} x^{r_{k}} x^{n}\left\{\xi_{1 k}+\frac{\xi_{1 k}^{(1)}}{x}+\cdots\right\}
$$

with respect to $x$ in any left half plane.

By means of (32) we now obtain the asymptotic formulas for $g_{2 k}^{\prime}(x), \ldots$, $g_{n k}^{\prime}(x)$. Thus we obtain $n$ formal expansions which must of course be the constituent elements of some formal solution. The possibility that $\xi_{1 / k}, \xi_{1 k}^{(1)}, \ldots$ are all zero must be excluded since from that fact we should infer that all the coefficients in the asymptotic expansion of $g_{i k}^{\prime}(x)(i=1,2, \ldots, n)$, and consequently 
of $\theta^{(k)}(x)$, were zero. The formal solution thus obtained must coincide with $s_{1 k}(x), \cdots, s_{n k}(x)$ at least if $\rho_{1}, \cdots, \rho_{n}$ are distinct. For it is apparent that the only possible difference lies in a constant factor. By definition of $\theta^{(k)}(x)$ in terms of $g_{11}(x)=g_{11}^{\prime}(x), \ldots, g_{1 k}=g_{1 k}^{\prime}(x)$, and in virtue of the fact that the asymptotic form of $\theta^{(k)}(x)$ has been shown to be that obtained by replacing these functions by $s_{11}(x), \ldots, s_{1 k}(x)$, it follows that this factor must be 1 . Hence we have in this case $\xi_{i k}=s_{i k}, \xi_{i k}^{(1)}=s_{i k}^{(1)}, \ldots$ Under our restriction, these equations are algebraic identities persisting in the exceptional cases when $\rho_{1}, \cdots$, , n $_{n}$ are not distinct also.

Thus we have demonstrated the existence of a $k$ th solution having the desired properties provided that $\left|\rho_{k-1}\right|$ and $\left|\rho_{k}\right|$ are not equal.

We shall now indicate briefly the modifications that are necessary in the case $\left|\rho_{k-1}\right|=\left|\rho_{k}\right|$. Suppose that $\rho_{k}$ is one of a group of roots

$$
\rho_{a}, \rho_{a+1}, \cdots, \rho_{\beta}
$$

of equal absolute values. If $\rho_{k}=\rho_{a}$, we have seen how to construct the $k$ th solution; if $\rho_{k}=\rho_{a+1}$, one may invert the order of $\rho_{a}$ and $\rho_{a+1}$ in (8) and thus construct a $(k+1)$ th solution possessing the requisite asymptotic properties, and so continue to the $\beta$ th solution. It is however necessary to know that the solutions thus constructed are associated with $u_{i}(x), u_{i j}(x), \ldots$ as stated. According to the method used in $\S 2$ there certainly exist $\alpha$ th, $(\alpha+1)$ th, $\ldots, \beta$ th solutions over congruent sets of points which satisfy all the limit determinant relations, obtained by interchanging the order of $\rho_{a}, \ldots, \rho_{\beta}$ in (8); for the algebraic condition that this hold will be satisfied at all the approximations since these are actual determinants, and hence will be satisfied in the limit also. On this account at least certain determinations of the sum formula employed will give a $k$ th associated solution. Since however this $k$ th associated solution is determined up to a linear combination with periodic coefficients of the solutions before the ath, and the formula which gives it indicates a solution determined up to a linear combination with periodic coefficients of solutions before the ath, the general solution given by the sum formula is a $k$ th associated solution. Thus the solutions, obtained in this exceptional case as indicated, are associated with the determinant limits.

It would be of considerable interest to determine the nature of these intermediate solutions near to the axis of reals.

Considerations of symmetry show that there exists a set of solutions $h_{1 j}^{\prime}(x)$, $\ldots, h_{n j}^{\prime}(x)(j=1,2, \ldots, n)$ having the properties stated.

\section{§4. The First and Second Principal niatrix Solutions.}

We shall proceed now to prove the existence of certain two remarkable matrix solutions $G(x)$ and $H(x)$ of (4), and this will be done by means of the 
formulas of $\S 2$ and the intermediate solutions of $\S 3$. It is clear that in order to define any matrix $G(x)$ it is merely necessary to choose properly the operations $\sum_{t=x}$ which occur in (30). We shall use a contour integral to effect these summations, similar to that which Carmichael has used, writing

$$
\sum_{t=x} \phi(t)=-\int_{L} e^{2 \pi \lambda v \overline{-1}(x-t)} \frac{\phi(t) d t}{e^{2 \pi v^{\prime}-1(x-t)}-1}
$$

where $\lambda$ is an integer and $L$ is a path $\infty A B \infty$ like that in fig. 2. The contour $A B$ is a simple curve which passes between $x$ and $x-1$. The summa-

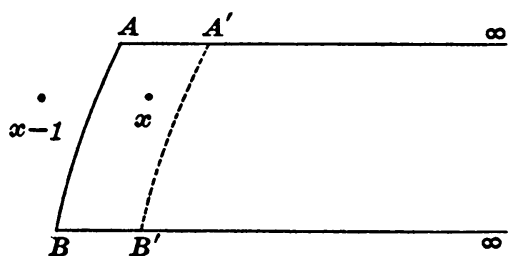

Fia. 2. tion function $\phi(t)$ is supposed to be analytic except at points at a limited distance from the positive axis of reals and within the region $\infty A B \propto$. Furthermore $\phi(t)$ is supposed to tend toward zero along lines $A \infty, B \infty$ sufficiently distant from the axis of reals, and in such manner as to make the above integral absolutely and uniformly convergent in the vicinity of any $x$ not congruent to a point of $L$. In this case the function represented by the integral will be analytic at a sufficient distance from the positive axis of reals, and will be independent of the particular lines $A \infty, B \infty$ or particular curve $A B$ chosen. If we form the difference $\sum_{t=x+1} \phi(t)-\sum_{t=x} \phi(t)$ it will be given by an integral over $A B B^{\prime} A^{\prime} A$, the integrand being unchanged. But within this closed curve the integrand is analytic save for a pole at $t=x$; and by the calculus of residues this difference reduces to $\phi(s)$. Hence this is a possible determination of $\sum_{t=x} \phi(t)$.

Having made this preliminary study of the operation $\sum_{t=x}$, we now proceed to the proof of

Theorem III. There exists a matrix solution $G(x)[H(x)]$ of $(4)$ whose elements have the following properties :

(a) the functions $g_{i j}(x)\left[h_{i j}(x)\right]$ satisfy the conditions

$$
\begin{gathered}
g_{i 1}(x)=u_{i}(x),\left|\begin{array}{ll}
g_{i 1}(x) & g_{i 2}(x) \\
g_{j 1}(x) & g_{j 2}(x)
\end{array}\right|=u_{i j}(x), \ldots \\
{\left[h_{i n}(x)=v_{i}(x),\left|\begin{array}{ll}
h_{i, n-1}(x) & h_{i n}(x) \\
h_{j, n-1}(x) & h_{j n}(x)
\end{array}\right|=v_{i j}(x), \ldots\right] ;}
\end{gathered}
$$

(b) the functions $g_{i j}(x)\left[h_{i j}(x)\right]$ are analytic throughout the finite plane except possibly for poles to the right (left) of and congruent to the poles of the elements of $A(x)\left[A^{-1}(x-1)\right]$;

(c) in any left (right) half plane $g_{i j}(x)\left[h_{i j}(x)\right]$ is asymptotically represented by $\boldsymbol{s}_{i j}(x)$ with respect to $x$. 
Proof. The proof of this theorem will be made by actually obtaining the solutions $g_{11}(x), \cdots, g_{n 1}(x) ; \cdots ; g_{1 n}(x), \cdots, g_{n n}(x)$ in succession. The conditions of the theorem are evidently satisfied by taking the first solution to be

$$
g_{11}(x)=u_{1}(x), \cdots, g_{n 1}(x)=u_{n}(x) .
$$

It suffices therefore to exhibit a solution $g_{1 k}(a), \cdots, g_{n k}(x)$ which satisfies the conditions of the theorem under the hypothesis that the solutions $g_{1 j}(x)$, $\cdots, g_{n j}(x)(j<k)$ have been constructed satisfying all of these conditions. This is the way in which the theorem will be demonstrated.

The formula (30) gives the most general determination of $g_{1 k}(x)$ which is possible if $g_{1 k}(x)$ is to be the first member of a kth solution satisfying restriction $(a)$. We shall consider first the case $\left|\rho_{k-1}\right|>\left|\rho_{k}\right|$. We choose the operation $\sum_{t=x}$ as indicated above, and obtain

$$
g_{1 k}(x)=-\sum_{j=1}^{k-1} g_{1 j}(x) \int_{L} e^{2 \pi \lambda_{j k} k^{\prime}-1}(x-t) \frac{\theta^{(k)}(t) m_{j, k-1}(t)}{\theta^{(k-1)}(t) \theta^{(k-1)}(t+1)} \frac{d t}{e^{2 \pi !-1}(x-t)}-1
$$

the integer $\lambda_{j k}$ will be chosen to be the least integer as great as the real part of

$$
\frac{1}{2 \pi 1-1}\left(\log \rho_{k}-\log \rho_{j}\right) \text {. }
$$

The functions $g_{2 k}(x), \cdots, g_{n k}(x)$ are then obtained from (32).

The first step is to establish the fact that the summation functions in (39) satisfy the restriction on the summation function $\phi(t)$. Now $\theta^{(k)}(x), \theta^{(k-1)}(x)$ are analytic save at points to the right of and congruent to the poles of elements $a_{i j}(x)$ by (25). Likewise, by the initial hypothesis and by definition of $m_{j, k-1}(x)$ as the cofactor of the element in the $j$ th column and last row of $\theta^{(k-1)}(x+1)$ [see the form (29)], the singularities of $n_{j, k-1}(x)(j=1,2$, $\ldots, k-1)$ are restricted in the same way. Hence the summation function

$$
\phi(t)=\frac{\theta^{(k)}(t) m_{j, k-1}(t)}{\theta^{(k-1)}(t) \theta^{(k-1)}(t+1)}
$$

will be analytic at all points sufficiently far from the positive axis of reals, provided that $\theta^{(k-1)}(t)$ does not vanish except at points within a certain distance of that axis. This fact is an immediate consequence of the relation (33) in which $d_{k}, d_{k}^{(1)}, \ldots$ have been seen not, all to be zero. It remains to show that the function $\phi(t)$ tends to zero in such a way that the integrals appearing in (39) converge as stated for all positions of the lines $A \infty$ and $B \infty$ at a sufficient distance from the axis of the reals. In order to demonstrate this essential fact we make use of the intermediate solutions obtained in $\S 3$. By Theorem II the general determination of the solutions $g_{1 j}(x), \cdots, g_{n j}(x)$ associated with 
the determinant limits is given by any one of them plus arbitrary periodic multiples of the preceding ones. Hence we have

$$
\begin{array}{r}
g_{i j}(x)=g_{i 1}^{\prime}(x) p_{1 j}(x)+\cdots+g_{i, j-1}^{\prime}(x) p_{j-1, j}(x)+g_{i j}^{\prime}(x) \\
(i=1,2, \cdots, n ; j=1,2, \cdots, k-1),
\end{array}
$$

where the functions $p_{i j}(x)$ are periodic functions of period 1, analytic for $x$ sufficiently distant from the axis of reals since the determinant $\left|g_{i j}^{\prime}(x)\right| \sim\left|s_{i j}(x)\right|$ does not then vanish. Substitute these expressions (41), for $i=1$, in $m_{j, k-1}(t)$. A reference to (29) makes it clear that the $m_{j, k-1}(t)$ may be written as a sum of products of periodic multipliers analytic for $x$ sufficiently distant from the axis of reals and of factors $m_{j, k-1}^{\prime}(t)$, where $m_{j, k-1}^{\prime}$ signifies as before that which $m_{j, k-1}$ becomes when $g^{\prime}$ is put in place of $g$. Hence the integrals will converge in the manner desired if the expressions

$$
\frac{\theta^{(k)}(t) m_{j, k-1}^{\prime}(t)}{\theta^{(k-1)}(t) \theta^{(k-1)}(t+1)}
$$

tend to zero in exponential fashion along $A \infty$ and $B \infty$. This is true if $A \infty$ and $B \infty$ are sufficiently distant from the axis of reals, in view of the asymptotic relationship (36) which holds with respect to $v$ in the right half plane.

The function $g_{1 k}(x)$ is therefore analytic at a sufficient distance from the positive axis of reals, and by (32) the same may be said of the remaining elements $g_{2 k}(x), \ldots, g_{n k}(x)$ which constitute the $k$ th solution. Working to the right across the plane by the aid of the original equations one proves readily that the only possible singularities of the functions of this solution will be poles congruent to the poles of the elements of $A(x)$ and to the right of them. Hence $(b)$ is true for the $k$ th solution. Also $(a)$ holds in so far as it relates to this solution since every solution $g_{1 k}(x), \cdots, g_{n k}(x)$ determined by (30) had this property.

Statement (c) has to do with the asymptotic nature of $g_{1 k}(x), \cdots, g_{n k}(x)$. In order to prove $(c)$ let us break up each integral in (39) into two others. over contours $L_{1}$ and $L_{2}$ as follows : the contour $L_{1}$ is a fixed contour $\infty A_{1} B_{1} \infty$ (fig. 3). If $x$ lies above $A_{1} \infty, L_{2}$ consists of a loop-circuit to $\infty$ which includes the points $x, x+1, \ldots$ within it but not $x-1, x-2, \ldots$ If $x$ lies between $A_{1} \infty$ and $B_{1} \infty, L_{2}$ consists of a loop which includes $x, x+1, \cdots$, $x+l$ within it, where $x+l$ is the last of this series of points to the left of $A_{1} B_{1}$. If $x$ lies below $B_{1} \infty, L_{2}$ is a loop-circuit of the same nature as when $x$ lies above $A_{1} \infty$. Any combination of paths $L_{1}$ and $L_{2}$ is clearly equivalent to the single path $\infty A B \infty$ of fig. 2. If $x$ lies on $A \infty$ or $B \infty$ or on a line congruent to a point $A_{1} B_{1}$ on the left, the two integrals are undefined.

Let us consider first the part of the integrals contributed by $L_{1}$. The integrands are periodic functions of $x$ of period 1 and therefore the integrals will 


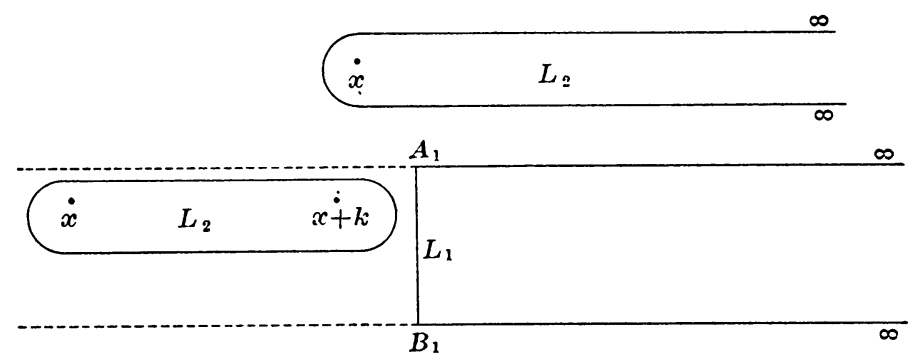

FIG. 3.

also be periodic since the contour $L_{1}$ is fixed. This part of the $j$ th one of these integrals may be written as either of the products

$$
\begin{aligned}
& e^{2 \pi \lambda_{j k} v^{-1} x} \int_{L_{1}} e^{-2 \pi \lambda_{j k} 1^{-1} t} \frac{\theta^{(k)}(t) m_{j, k-1}(t)}{\theta^{(i-1)}(t) \theta^{(k-1)}(t+1)} \frac{d t}{e^{2 \pi I^{\prime}-1(x-t)}-1}, \\
& e^{2 \pi\left(\lambda_{j k}-1\right) \sqrt{-1} x} \int_{L_{1}} e^{-2 \pi\left(\lambda_{j k}-1\right) 1^{\prime}-t} \frac{\theta^{(k)}(t) m_{j, k-1}(t)}{\theta^{(k-1)}(t) \theta^{(k-1)}(t+1)} \frac{d t}{1-e^{-2 \pi l-1(x-t)}} .
\end{aligned}
$$

It is apparent that in the first of these the second factor remains limited as $v$ becomes positively infinite, inasmuch as $e^{2 \pi \sqrt{-1} x}$ tends toward zero; likewise that in the second of these the second factor remains limited as $v$ becomes negatively infinite. Hence the part of $g_{1 k}$ thus obtained is

$$
C_{L_{1}}=\left\{\begin{array}{l}
g_{11}(x) e^{2 \pi \lambda_{1 k} V-1 x} q_{1 k}(x)+\cdots+g_{1, k-1}(x) e^{2 \pi \lambda_{k-1, k} v^{\gamma-1} x} q_{k-1, k}(x), \\
g_{11}(x) q_{1 k}(x)+\cdots+g_{1, k-1}(x) q_{k-1, k}(x), \\
g_{11}(x) e^{2 \pi\left(\lambda_{1 k}-1\right) v^{\prime}-1 x} q_{1 i}(x)+\cdots+g_{1, k-1}(x) e^{2 \pi\left(\lambda_{k-1, k}-1\right) \gamma^{\prime}-1 x} q_{k-1, k}(x),
\end{array}\right.
$$

according as $x$ lies above $A_{1} \infty$, between $A_{1} \infty$ and $B_{1} \infty$, or below $B_{1} \infty$. The functions $q_{1 k}(x), \ldots, q_{k-1, k}(x)$ are periodic and limited. This is obviously true unless $x$ is in the vicinity of a point congruent to a point of the contour of integration. However it is true in this case also since each of the three expressions above is unchanged if the contour be varied continuously; of course the function $q_{i k}(x)$ is not one and the same analytic function in the three cases.

Next let us consider the contribution $C_{L_{2}}$ yielded by the integrals taken over $L_{2}$. Let us suppose first that $x$ lies above $A_{1} \infty$ or below $B_{1} \infty$. In this event the integrals in $C_{L_{2}}$ may be evaluated as a sum of residues, each of which may be expressed in two ways, as indicated in the following formulas

$$
C_{L_{2}}=-\sum_{j=1}^{k-1} g_{1 j}(x) \sum_{\nu=0}^{\infty}\left[\frac{\theta^{(k)}(x+\nu) m_{j, k-1}(x+\nu)}{\theta^{(k-1)}(x+\nu) \theta^{(k-1)}(x+\nu+1)}\right]
$$

or

$$
C_{L_{2}}=-\sum_{j=1}^{k-1} g_{1 j}(x) \sum_{\nu=0}^{\infty}\left[\frac{\theta^{\left(k_{i}\right)}(x+\nu) m_{j, k-1}^{\prime}(x+\nu)}{\theta^{(k-1)}(x+\nu) \theta^{(k-1)}(x+\nu+1)}\right]
$$


The first form is the obvious one derived from equation (39). If we observe however that we have, by definition of $m_{j, k-1}(x)$,

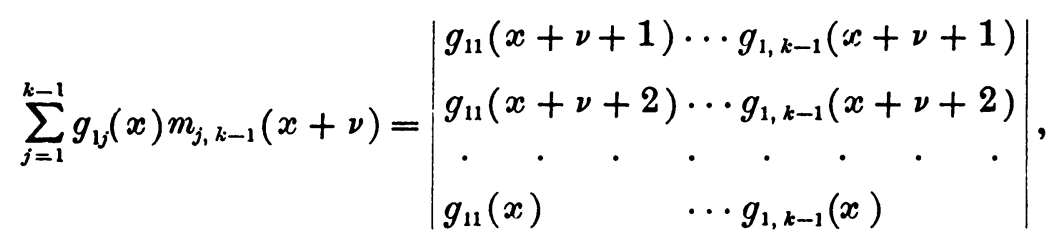

and then write in place of $g_{1 j}$ their equals as given in (41), the resulting determinant may be simplified because the periodic coefficients are the same at $x+\nu+1, x+\nu+2, \ldots$ as at $x$. By a combination of successive columns the determinant reduces to a new determinant identical with the above except that $g^{\prime}$ replaces $g$, i. e., reduces to

$$
\sum_{j=1}^{k-1} g_{1 j}^{\prime}(x) h_{j, k-1}^{\prime}(x+\nu) \text {. }
$$

Thus the second of the above forms is obtained except that the two signs of summation will appear in inverted order. It is permissible to change this order if all the infinite series converge. This is certainly the case as the second form is precisely that which defined $g_{1 k}^{\prime}(x)$ in $\S 3$ [see (34)]. Thus we have for $x$ above $A_{1} \infty$ or below $B_{1} \infty$

$$
C_{L_{2}}=g_{1 k}^{\prime}(x) \text {. }
$$

When $x$ lies between $A_{1} \infty$ and $B_{1} \infty$ to the left of $A_{1} B_{1}$ we use a form like (43). We will agree to denote $C_{L_{2}}$ by $g_{1 k}^{\prime}(x)$ for the sake of convenience, because then (44) holds in all cases and $g_{1 k}^{\prime}(x)$ as thus defined will have the asymptotic form $8_{1:}$ with respect to $x$ in any left half plane, and u:ith respect to $v$ in any right half plane. In $\S 3$ these facts have already been proved for $g_{1 k}^{\prime}(x)$ as there defined, that is for $x$ not between $A_{1} \infty$ and $B_{1} \infty$. We need therefore to prove that $g_{1 k}^{\prime}(x) \sim s_{1 k}(x)$ with respect to $x$ between $A_{1} \infty$ and $B_{1} \infty$ only. The discussion is entirely similar to a discussion that has been given in $\S 3$. In the first place the integrals in the expression $g_{1 k}^{\prime}(x)$ for $C_{L_{2}}$ may be evaluated as a sum of residues

$$
g_{1 k}^{\prime}(x)=-\sum_{j=1}^{k-1} g_{1 j}(x) \sum_{\nu=11}^{l} \frac{\theta^{(k)}(x+\nu) m_{j, k-1}(x+\nu)}{\theta^{(k-1)}(x+\nu) \theta^{(k-1)}(x+\nu+1)},
$$

where $x+l$ is the last point of the set $x, x+1, \ldots$ to the left of $A_{1} B_{1}$. If this equation be compared with (34) one sees that the right hand members differ in that a finite sum replaces an infinite series. Proceeding precisely as we did with (34) we arrive at the asymptotic relation $g_{1 k}^{\prime}(x) \sim s_{1 k}(x)$ with respect to $x$ by means of the hypothesis made that $g_{1 j}(x), \cdots, g_{n j}(x)$ are asymptotically represented by $s_{1 j}(x), \cdots, s_{n j}(x)$ with respect to $x$ in any left half plane and 
for $j=1,2, \cdots, k-1$. The sole modification consists in this that instead of having an infinite set of terms in which those after the $m$ th ( $m$ the greatest integer less than $\frac{1}{2}|x|$ ) do not affect the asymptotic form, one has only a finite set of terms in which however those after the $m$ th do not affect the asymptotic form.

The proof of $(c)$ of the theorem in so far as it relates to $g_{1 k}(x), \cdots, g_{n k}(x)$ may now be given. In the first place, for $x$ above $A_{1} \infty,(42)$ and (44) give

$$
g_{1 k}(x) \sim s_{11}(x) e^{2 \pi \lambda_{1 k} \sqrt{-1} x} q_{1 k}(x)+\cdots+s_{1, k-1}(x) e^{2 \pi \lambda_{k-1, k} \sqrt{-1} x} q_{k-1, k}+s_{1 k}(x) .
$$

The dominating term will depend upon the dominating term of

$$
e^{\left(2 \pi \lambda_{1 k} \sqrt{-1}+\log \rho_{1}\right) x}, \cdots, e^{\left(2 \pi \lambda_{k-1}, k v^{\prime} \overline{-1}+\log \rho_{k-1}\right) x}, e^{\left(\log \rho_{k}\right) x}
$$

which begin the formal expansions. On dividing by the last of these, the exponents become

$2 \pi \sqrt{-1}\left(\lambda_{1 k}+\frac{\log \rho_{1}-\log \rho_{k}}{2 \pi V-1}\right) x, \ldots, 2 \pi \sqrt{-1}\left(\lambda_{k-1, k}+\frac{\log \rho_{k-1}-\log \rho_{k}}{2 \pi \sqrt{-1}}\right) x, 0$.

The bracketed expressions here all have a positive real part less than $1:$ this follows from the definition of $\lambda_{1 k}, \cdots, \lambda_{k-1, k}$ and the fact that $\left|\rho_{k-1}\right|>\left|\rho_{k}\right|$. Hence the coefficients of $x$ are represented by points in the upper half plane below the line $v=2 \pi$. Therefore if $x$ lies in the second quadrant, all of these exponents have a negative real part, and the last term dominates, that is

$$
g_{1 k}(x) \sim s_{1 k}(x)
$$

for $x$ above $A_{1} \infty$ and to the left of the axis of imaginaries, or likewise of any parallel line.

For $x$ between $A_{1} \infty$ and $B_{1} \infty$ we have similarly

$$
g_{1 k}(x) \sim s_{11}(x) q_{1 k}(x)+\cdots+s_{1, k-1}(x) q_{k-1, k}(x)+s_{1 k}(x)
$$

and the last term clearly dominates again by (8).

For $x$ below $B_{1} \infty, g_{1 k}(x)$ is represented by

$$
s_{11}(x) e^{2 \pi\left(\lambda_{1 k}-1\right) \sqrt{-1} x} q_{1 k}(x)+\cdots+s_{1, k-1}(x) e^{-2 \pi\left(\lambda_{k-1, k-1)} \sqrt{-1} x\right.} q_{k-1, k}(x)+s_{1 k}(x) .
$$

The dominating term depends on the exponents

$2 \pi \sqrt{-1}\left(\lambda_{1 k}-1+\frac{\log \rho_{1}-\log \rho_{k}}{2 \pi \sqrt{-1}}\right) x, \ldots, 2 \pi \sqrt{-1}\left(\lambda_{k-1, k}-1+\frac{\log \rho_{k-1}-\log \rho_{k}}{2 \pi \sqrt{-1}}\right) x, 0$.

The coefficients of $x$ will in this case clearly lie below the real axis and above the line $v=2 \pi$, and if $x$ lies in the third quadrant, the last term $s_{1 k}(x)$ again dominates.

Hence in any left half plane $g_{1 k}(x)$ is asymptotically represented by $s_{1 k}(x)$ 
with respect to $x$, and of course $g_{2 k}(x), \ldots, g_{n k}(x)$ are at the same time represented by $s_{2 k}(x), \ldots, s_{n k}(x)$ respectively, as follows from (32). Thus a solution $g_{1 k}(x), \cdots, g_{n k}(x)$ exists possessing properties $(a),(b),(c)$ unless $\left|\rho_{k-1}\right|=\left|\rho_{k}\right|$, that is unless $\rho_{k}$ is one of a group $\rho_{a}, \rho_{a+1}, \cdots, \rho_{p}$ of roots $\rho$ of equal absolute value. The same argument which enabled us to treat this exceptional case in $\S 3$ may now be applied here; that is, we may take $\rho_{k}$ as the first of this group without violating (8) and construct a $k$ th solution which satisfies $(a),(b),(c)$ corresponding to each of these values of $\rho$.

The second set of solutions $h_{1 j}(x), \cdots, h_{n j}(x),(j=1,2, \cdots, n)$ may be obtained by considerations of symmetry.

There is one and only one solution $g_{1 k}(x), \ldots, g_{n k}(x)\left[h_{1 k}(x), \ldots, h_{n k}(x)\right]$ $(k=1,2, \cdots, n)$ analytic except for poles and asymptotically represented by $s_{1 k}(x), \cdots, s_{n k}(x)$ even to terms of the first order in any left (right) half plane. The matrix $G(x)[H(x)]$ with elements $g_{i j}(x)\left[h_{i j}(x)\right]$ is termed the first (second) principal matrix solution of (4).

In order to justify this definition it is necessary to show that there exists but one such solution $g_{1 k}(x), \cdots, g_{n k}(x)\left[h_{1 k i}(x), \cdots, h_{n k}(x)\right]$. Suppose if possible that there exists a second solution $\bar{g}_{1 k}(x), \cdots, \bar{g}_{n k}(x)$ with the properties stated. Now we can express $\bar{g}_{i k}(x)$ as follows :

$$
\bar{J}_{i k}(x)=g_{i 1}(x) p_{1}(x)+\cdots+g_{i n}(x) p_{n}(x) \quad(i=1,2, \cdots, n),
$$

where $p_{1}(x), \cdots, p_{n}(x)$ are periodic of period 1 , inasmuch as $g_{1 j}(x), \cdots$, $g_{n j}(x)(j=1,2, \cdots, n)$ form a fundamental system of solutions. If we solve these equations for $p_{i}(x)$, we shall have

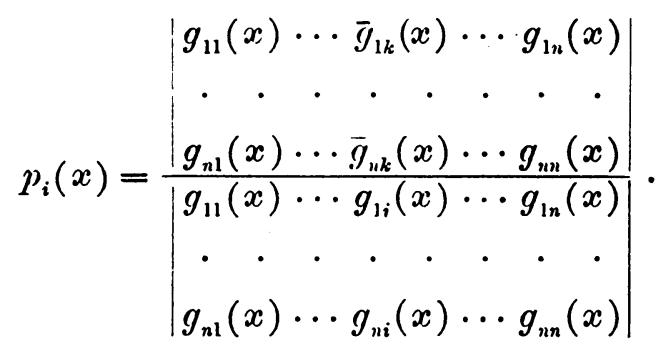

Consider now some definite period-strip formed by two lines parallel to the axis of imaginaries and one unit apart, and let $x$ become infinite in this strip. On account of the known asymptotic form of the elements $g$ and $\bar{g}$ in the strip to terms of the first order we find at once, if $i \neq k$,

$$
p_{i}(x) \sim\left(\frac{\rho_{k}}{\rho_{i}}\right)^{x} x^{r_{k}-r_{i}}[0]
$$

for $x$ at either end of the strip. If we write $z=e^{2 \pi \sqrt{-1} x}$, this may be written

$$
p_{i}(x) \sim z^{\frac{1}{2 \pi \sqrt{-1}}\left(\log \rho_{k}-\log \rho_{i}\right)}(\log z)^{r_{k}-r_{i}}[0] .
$$


The upper end of the strip corresponds to $z=0$, and the lower end to $z=\infty$. The above relation shows that $p_{i}(x)$ has a zero of at least the order $\lambda_{i k}$ at $z=0$. Likewise, this relation shows that $p_{i}(x)$ has a pole of order $\lambda_{i k}-1$ at most at $z=\infty$. Consequently $p(x) z^{-\lambda_{i k}}$ is analytic at $z=0$ and vanishes at $z=\infty$. Moreover, by taking $x$ far enough to the left, the original determinant expression for $p_{i}(x)$ is analytic since every element of the two determinants is analytic, and the denominator is asymptotic to the formal determinant $\left|s_{i j}(x)\right|$ which is not zero if $x$ lies far to the left. Hence $p_{i}(x) z^{-\lambda_{i k}}$, for $i \neq k$, is analytic for all finite values of $z$ and vanishes at $z=\infty$; it must be identically zero. We conclude that

$$
g_{i k}(x)=g_{i k}(x) p_{k}(x) .
$$

Now let $i$ be chosen so that $s_{i k}$, the constant of the principal term of $s_{i k}(x)$, is not zero, as is possible by (7). Consider any point $x_{0}$ and the points $x_{0}-1$, $x_{0}-2, \ldots$ to its left. As $x$ takes on these values, $\bar{g}_{1 k}(x) / g_{1 k}(x)$ tends toward 1 , and thus we see that $p_{k}(x)$ is 1 at the point $x_{0}$. That is, $\bar{g}_{1 k i}(x)$ and $g_{1 k}(x)$ are one and the same function, in contradiction to our hypothesis.

If the functions $a_{i j}(x)$ had not been restricted to be rational, the only modification in $\S \S 1-4$ would be that in the statement and proof of $(b)$, Theorem III, other singularities than poles would enter.

\section{§5. The Fundamental Periodic Functions.}

Either of the two principal matrix solutions $G(x)$ and $H(x)$ of (4) may be expressed in terms of the other, e. g.,

$$
G(x)=H(x) P(x),
$$

in which the elements $p_{i j}(x)$ of $P(x)$ are periodic functions of period 1 which may be termed the fundamental periodic functions. We shall now determine the nature of these functions. The fact that will enable us to do this is that $G(x)$ and $H(x)$ are composed of elements of known asymptotic form in any period strip which may be taken to be one and the same in both cases.

On account of the gain in simplicity we shall make a preliminary transformation. Let

$$
d(x)=(x-\alpha)(x-\beta) \cdots(x-\lambda)
$$

be the least common denominator of the set of rational functions $a_{i j}(x)$, and write

$$
\bar{g}_{i}(x)=\Gamma(x-\alpha) \Gamma(x-\beta) \cdots \Gamma(x-\lambda) g_{i}(x) \quad(i=1,2, \cdots, n),
$$

where $\Gamma(x)$ is the gamma function. The transformed system will be altered in that the common denominator has been removed. The formal solutions of the new system will be those of the old multiplied by a certain factor, and hence will satisfy the restrictions imposed in $\S 1$. 
The new coefficients are polynomials in $x$. It is then permissible to take all the functions $a_{i j}(x)$ as polynomials, say of degree $\mu$ or less, and this we shall do. The first principal matrix solution $G(x)$ is constituted of elements analytic throughout the finite plane, in this case, inasmuch as the elements $a_{i j}(x)$ have no poles in the finite plane.

From the equation (46) we have

$$
P(x)=H^{-1}(x) G(x) ;
$$

but if we take our period strip far enough to the right, the elements of $H(x)$ are analytic functions of $x$ and the determinant of $H(x)$ will not vanish in the strip inasmuch as its asymptotic form is given by the determinant $\left|s_{i j}(x)\right|$. Consequently the elements $p_{i j}(x)$ are functions of $z=e^{2 \pi \sqrt{-1} x}$ which are analytic except perhaps at $z=0$ and $z=\infty$, corresponding to the upper and lower end of the period strip. In the case when the functions $a_{i j}(x)$ in (1) are merely rational it is clear that the periodic functions $p_{i j}(x)$ are analytic or have poles except at $z=0$ or $z=\infty$.

It is necessary now to remove a certain ambiguity. The expression $s_{i j}(x)$ represents the elements $g_{i j}(x)$ and $h_{i j}(x)$ asymptotically in any right and left hand side of the plane respectively, and is a many-valued expression which is changed to

$$
e^{2 \pi \mu \sqrt{-1 x}} e^{2 \pi r_{j} \sqrt{-1}} s_{i j}(x)
$$

after a complete positive circuit of $x=0$ [see (6)]. Hence if the same determination of $s_{i j}(x)$ is chosen for $g_{i j}(x)$ and $h_{i j}(x)$ along the direction of the positive axis of imaginaries, the elements $g_{i j}(x)$ and $h_{i j}(x)$ will have a different asymptotic form along the direction of the negative axis of imaginaries, since in one case we pass from the positive axis of imaginaries to the negative by a positive partial circuit, and in the other by a negative partial circuit. (It must be remembered that $g_{i j}(x)$ and $h_{i j}(x)$ preserve their asymptotic form in any left and right half plane respectively.) Let the determination for $h_{i j}(x)$ be termed $s_{i j}(x)$. Then that of $g_{i j}(x)$ is obviously obtained by a complete positive circuit and is given by (48) in the direction of negative imaginaries.

In the upper half of the period strip $g_{i j}(x)$ and $h_{i j}(x)$ have a common asymptotic representation $s_{i j}(x)$, and this proves that

$$
P(x) \sim S^{-1}(x) S(x) .
$$

But the elements of $s_{i j}^{\prime}(x)$ and $s_{i j}(x)$ of $S^{-1}(x)$ and $S(x)$ are of the respective asymptotic forms

$$
x^{\mu x} \rho_{i}^{-x} x^{-r_{i}}\left\{\sigma_{i j}+\frac{\sigma_{i j}^{(1)}}{x}+\cdots\right\}, \quad x^{\mu x} \rho_{j}^{x} x^{r_{j}}\left\{s_{i j}+\frac{s_{i j}^{(1)}}{x}+\cdots\right\}
$$


[see (9) and (6)], so that in consequence the relation holds

$$
p_{i j}(x) \sim \rho_{i}^{-x} \rho_{j}^{x} x^{-r_{i}+r_{j}} \delta_{i j} .
$$

Therefore, if we think of $p_{i j}(x)$ as a function of $z$, we have

$$
\lim _{z=0} p_{i j}(x) z^{-\frac{\log \rho_{j}-\log \rho_{i}}{2 \pi \sqrt{-1}}}\left(\frac{\log z}{2 \pi \sqrt{-1}}\right)^{r_{i}-r_{j}}=\delta_{i j} .
$$

The single-valued analytic function $p_{i j}(x)$ of $z$ is accordingly infinite only to a finite order at $z=0$ and either has a pole there, or is analytic. For $i=j$ it is clear that $p_{i v}(x)$ is an entire function of $z$ taking on the value 1 at $z=0$, that is

$$
p_{i i}(x)=1+c_{i i}^{(1)} e^{2 \pi \sqrt{-1} x}+\cdots
$$

For $i \neq j$ we have obviously

$$
\lim _{z=0} p_{i j}(x) z^{-\lambda_{i j}}=\text { constant }
$$

since $\lambda_{i j}$ was defined as the least integer as great as the real part of

$$
\frac{\log \rho_{j}-\log \rho_{i}}{2 \pi \sqrt{-1}}
$$

Hence $p_{i j}(x)$ has a zero of order $\lambda_{i j}$, at least, at $z=0$, and may be written

$$
p_{i j}(x)=e^{2 \pi \lambda_{i j} \cdot \overline{-1} x}\left(c_{i j}^{(0)}+c_{i j}^{(1)} e^{2 \pi \sqrt{-1} x}+\cdots\right) \text {, }
$$

the coefficient of the exponential factor being an entire function of $z$.

It remains to consider the functions $p_{i j}(x)$ in the lower half of the period strip. Here the functions $g_{i j}(x)$ and $h_{i j}(x)$ have the respective asymptotic forms

$$
e^{2 \pi \mu \sqrt{-1} x} e^{2 \pi r_{j} \sqrt{-1}} s_{i j}(x) \text { and } s_{i j}(x),
$$

and the matrices $G(x)$ and $H(x)$ are asymptotically represented by

respectively where

$$
S(x) \text { and } S_{1}(x)
$$

Hence we obtain

$$
S_{1}(x)=S(x)\left(e^{2 \pi \mu \sqrt{-1} x} e^{2 \pi r_{j} \sqrt{-1}} \delta_{i j}\right) \cdot *
$$

$$
\begin{aligned}
& P(x) \sim S^{-1}(x) S_{1}(x) \text { or }\left[S^{-1}(x) S(x)\right]\left(e^{2 \pi \mu \sqrt{-1 x}} e^{2 \pi r_{j} \sqrt{-1}} \delta_{i j}\right) \\
& \text { or } \rho_{i}^{-x} \rho_{j}^{x} x^{-r_{i}+r_{j}}\left(e^{2 \pi \mu \sqrt{-1} x} e^{2 \pi r_{j} \sqrt{-1}} \delta_{i j}\right) \text {. }
\end{aligned}
$$

Thus each function $p_{i j}(x)$ satisfies the condition

$$
\lim _{z=\infty} p_{i j}(x) z^{-\mu-\frac{\log \rho_{j}-\log \rho_{i}}{2 \pi \sqrt{-1}}}\left(\frac{\log z}{2 \pi \sqrt{-1}}\right)^{r_{i}-r_{j}} e^{-2 \pi r_{j} \sqrt{-1}}=\delta_{i j} .
$$

* The notation $\left(\theta_{i j}\right)$ signifies a matrix with term $\theta_{i j}$ in the $i$ th row and $j$ th column. 
We see again that $p_{i j}(x)$ becomes infinite only to a finite order at $z=\infty$. This demonstrates that $p_{i i}(x)$ is a polynomial in $z$ and that $p_{i j}(x)(i \neq j)$ is equal to $z^{\lambda_{i j}}$ multiplied by a polynomial in $z$.

In the case $i=j$ we have

$$
\lim _{z=\infty} p_{i i}(x) z^{-\mu}=e^{2 \pi r_{i} \nu-1}
$$

so that the polynomial $p_{i i}(x)$ is of degree $\mu$ in $z$ with leading coefficient $e^{2 \pi r_{i}} \overline{j-1}$. In the case $i \neq j$ we have

$$
\lim _{z=\infty} p_{i j}(x) z^{-\mu-\lambda_{i j}+1}=\text { constant, }
$$

so that the polynomial coefficient of $z^{\lambda_{i j}}$ is of degree $\mu-1$.

Thus we have proved the following theorem :

Theorem IV. Let $G(x)$ and $H(x)$ be the first and second principal matrix solutions of (4) in which the elements of $H(x)$ are polynomials of degree $\mu$ at most in $x$, and write

$$
G(x)=H(x) P(x) .
$$

The fundamental periodic functions $p_{i j}(x)$ which constitute $P(x)$ are in this case of the form

$$
\begin{aligned}
& p_{i i}(x)=1+c_{i i}^{(1)} e^{2 \pi \sqrt{ }-1 x}+\cdots+e^{2 \pi r_{i} \mathcal{J}-1} e^{2 \pi \mu \sqrt{-1} x}, \\
& p_{i j}(x)=e^{2 \pi \lambda_{i j} \mathcal{J}-1 x}\left[c_{i j}^{(1)}+c_{i j}^{(1)} e^{2 \pi \sqrt{ }-1 x}+\cdots+c_{i j}^{(\mu-1)} e^{2 \pi(\mu-1) \sqrt{ }-1 x}\right] \quad(i \neq j),
\end{aligned}
$$

in which $\lambda_{i j}$ denotes the least integer as great as the real part of

$$
\frac{1}{2 \pi v^{\prime}-1}\left(\log \rho_{j}-\log \rho_{i}\right) \text {. }
$$

The simplest illustration is given by the difference equation for the Gamma function, when one has

$$
g(x)=e^{\pi \sqrt{-1} x-2 \pi \sqrt{-1}} \overline{\Gamma(1-x)}, h(x)=\Gamma(x), p(x)=1-e^{2 \pi^{v-1} x} .
$$

A similar analysis of the nature of the functions $p_{i j}(x)$ at $z=0$ and $z=\infty$ may be made in the more general case when the functions $a_{i j}(x)$ are not restricted to be rational functions, and shows that $p_{i j}(x)$ is analytic or has a pole at $z=0$ and $z=\infty$ with leading terms of the same form as those of (51) at these points. If the $a_{i j}(x)$ are rational, it is obvious that the functions $p_{i j}(x)$ are rational in $e^{2 \pi} \sqrt{-1 x}$; we shall not stop to investigate further the structure of these functions. 


\section{§6. The Principal Matrix Solutions in the Entire Plane.}

The results of the preceding paragraph make it possible to discuss the asymptotic form of the elements of $G(x)$ [and likewise of $H(x)$ ] in the entire complex plane. In the first place it has been shown that

$$
g_{i j}(x) \sim s_{i j}(x)
$$

along any ray from $x=0$ in the second or third quadrants. To investigate the asymptotic form along rays in the first and fourth quadrants we make use of the equation

$$
G(x)=H(x) P(x) \quad \text { or } \quad g_{i j}(x)=\sum_{\tau=1}^{n} h_{i \tau}(x) p_{\tau j}(x) \quad(i, j=1,2, \cdots, n),
$$

in which the elements $h_{i j}(x)$ are of known asymptotic form $s_{i j}(x)$ in any right half plane, and the elements $p_{i j}(x)$ are known functions of $x$.

Consider now a ray from $x=0$ in the first quadrant and lying above the axis of reals. Along this ray, by equations (51), we shall have

$$
p_{i j}(x) \sim e^{2 \pi \lambda_{i j} \sqrt{-1} x} c_{i j}^{(0)}, \quad \text { where } \lambda_{i i}=0, \quad c_{i i}^{(0)}=1 .
$$

Thus the preceding equation for $g_{i j}(x)$ leads to

$$
g_{i j}(x) \sim s_{i 1}(x) e^{2 \pi \lambda_{1 j} \sqrt{ }-1} c_{1 j}^{(0)}+\cdots+s_{i n}(x) e^{2 \pi \lambda_{n j} \sqrt{ }-1 x} c_{n j}^{(0)},
$$

a formula which determines the asymptotic form in the first quadrant.

To determine the dominant term we need to compare the relative magnitude of the terms

$$
\rho_{1}^{x} e^{2 \pi \lambda_{1} j \sqrt{-1} x}, \cdots, \rho_{j}^{r}, \cdots, \rho_{n}^{r} e^{2 \pi \lambda_{n j} \sqrt{-1} x} .
$$

If these be divided by $\rho_{j}^{r}$, the logarithms become

$$
\begin{aligned}
2 \pi V-1 & \left(\frac{\log \rho_{1}-\log \rho_{i}}{2 \pi l-1}+\lambda_{1 j}\right) x, \ldots, 0 x, \ldots, \\
& 2 \pi \sqrt{-1}\left(\frac{\log \rho_{n}-\log \rho_{j}}{2 \pi \sqrt{-1}}+\lambda_{n j}\right) x .
\end{aligned}
$$

By the very definition of $\lambda_{i j}$, the coefficients $\epsilon_{1 j}, \ldots, \epsilon_{j j}=0, \ldots, \epsilon_{n j}$ of $x$ in these expressions will lie in the strip $0 \leqq v<2 \pi$. Let $P_{1}, \ldots, P_{n}$ be the points which represent these coefficients in the complex plane. On account of (8) these coefficients will have decreasing real parts and the points $P_{1}, \ldots, P_{n}$ are ordered from right to left. As arg $x$ increases from 0 to $\frac{1}{2} \pi$ the above logarithms maintain their relative positions but are rotated about $x=0$ through the same angle. The dominant term along the ray $\arg x=\tau$ is that one for which the corresponding point is farthest to the right (i. e., the logarithm has the greatest real part) when a rotation $\tau$ has been effected. None of the terms after the $j$-th 
can dominate since the corresponding points are to the left of $P_{j}=0$ for all values of $\tau<\pi / 2$.

Draw the convex broken line $P_{1} P_{\sigma} \ldots P_{j}$ below which none of the points $P_{1}, \ldots, P_{j}$ lie and whose vertices are at some of these points. If the plane be rotated through an angle $\tau$, it is clear that these vertex-points, and only these, will correspond to dominant terms. Let the acute angles which the successive sides of $P_{1} P_{\sigma} \ldots P_{j}$ make with the axis of reals be $\phi_{1}, \phi_{\sigma}, \ldots$ The critical rays along which the dominant term changes are then in order of increasing magnitude

$$
\frac{\pi}{2}--\phi_{1}, \frac{\pi}{2}-\phi_{\sigma}, \cdots
$$

For small values of arg $x$ the dominant term is clearly given by

$$
\boldsymbol{s}_{i 1}(x) e^{2 \pi \lambda_{1 j} \sqrt{--1} x} c_{1 j}^{(0)},
$$

and as the first critical ray is passed this changes to

$$
s_{i \sigma}(x) e^{2 \pi \lambda_{\sigma j} \sqrt{-1} x} c_{\sigma j}^{(0)} \text {. }
$$

Along the ray the form is given by a combination of two or more terms, a fact which we shall express by saying that the change is uniform along the ras. A similar change takes place along each of the successive critical rays until the last is passed when the asymptotic form becomes $s_{i j}(x)$.

Likewise in the fourth quadrant the asymptotic nature is deduced from $a$ relation

$$
\begin{aligned}
g_{i j}(x) \sim s_{i 1}(x) e^{2 \pi\left(\mu+\lambda_{1 j}-1\right) \sqrt{-1} x} c_{1 j}^{(\mu-1)}+\cdots & +s_{i j}(x) e^{2 \pi \mu \sqrt{-1} x} e^{2 \pi r_{j} \sqrt{-1}} \\
& +\cdots+s_{i n}(x) e^{2 \pi\left(\mu+\lambda_{N j}-1\right) \sqrt{-1} x} c_{n j}^{(\mu-1)} .
\end{aligned}
$$

The expressions $s_{i 1}(x), \ldots, s_{i n}(x)$ which appear here are the determinations for the functions $h_{i 1}(x), \cdots, h_{i n}(x)$ in the right half plane. Consequently the determination of $s_{i j}(x)$ obtained by a positive rotation from the first to the fourth quadrant along the negative axis of reals is this determination multiplied by the: factor $e^{2 \pi \mu \sqrt{-1 x}} e^{2 \pi r_{j} \sqrt{-1}}$. Hence in terms of the second determination

$$
\begin{aligned}
g_{i j}(x) \sim s_{i 1}(x) e^{2 \pi\left(\lambda_{1 j}-1\right) \sqrt{-1} x} e^{-2 \pi r_{j} \sqrt{-1}} c_{1 j}^{(\mu-1)} & +\cdots+s_{i j}(x) \\
& +\cdots+s_{i n}(x) e^{2 \pi\left(\lambda_{n j}-1\right) V \overline{-1} x} e^{-2 \pi r_{j} V-1} c_{n j}^{(\mu-1)} .
\end{aligned}
$$

After division the corresponding logarithms are

$$
\begin{aligned}
2 \pi v-1\left[\frac{\log \rho_{1}-\log \rho_{j}}{2 \pi \sqrt{-1}}+\lambda_{1 j}-1\right] & x, \ldots, 0 x, \ldots, \\
& 2 \pi v-1\left[\frac{\log \rho_{n}-\log \rho_{j}}{2 \pi v-1}+\lambda_{n j}-1\right] x .
\end{aligned}
$$


The coefficients of $x$ are represented by points $Q_{1}, \ldots, Q_{j}=0, \ldots, Q_{n}, 2 \pi$ units below the corresponding points $P_{1}, \ldots, P_{n}$ (except for $P_{j}=Q_{j}=0$ ). These points all lie in the strip $-2 \pi \leqq v<0$ and are given in order from right to left. As arg $x$ decreases from 0 to $-\pi / 2$, the figure formed by these points rotates through an angle $-\pi / 2$, and the dominant terms correspond to the point farthest to the right. None of the terms after the $j$ th can dominate.

In this case we are led to the following rule: draw a convex broken line $Q_{1} Q_{\theta} \ldots Q_{j}$ above which none of the points $Q_{1}, Q_{2}, \ldots, Q_{j}$ lie and whose vertices are at these points. Let the acute angle which the successive sides $Q_{1} Q_{\theta} \ldots Q_{j}$ make with the axis of $x$ be $\psi_{1}, \psi_{\theta}, \ldots$ The critical rays along which the dominant term changes are then

$$
-\frac{\pi}{2}+\psi_{1},-\frac{\pi}{2}+\psi_{\theta}, \cdots
$$

in order of decreasing magnitude. For small $\arg x$ the dominant term has the asymptotic form

$$
\boldsymbol{s}_{i 1}(x) e^{2 \pi\left(\lambda_{1 j}-1\right)^{V-1} x} e^{-2 \pi r_{j}{ }^{V-1}} c_{1 j}^{(\mu-1)},
$$

and as the first critical ray is passed, this changes uniformly to

$$
s_{i \theta}(x) e^{2 \pi\left(\lambda_{\theta j}-1\right)^{v-1} \bar{x}} e^{-2 \pi r_{\theta} \overline{-1}^{-1}} c_{\theta_{j}}^{(\mu-1)},
$$

and similar changes take place along the remaining critical rays until the last ray is passed, when the form is $s_{i j}(x)$.

THEOREM V. Let the coefficients $a_{i j}(x)$ be polynomials of degree not greater than $\mu$ in $x$. Write

$$
2 \pi \sqrt{-1}\left(\frac{\log \rho_{i}-\log \rho_{j}}{2 \pi \sqrt{-1}}+\lambda_{i j}\right)=\epsilon_{i j}
$$

where $\lambda_{i j}$ is the least integer as great as the real part of the negative of the first term in parenthesis, and where $\rho_{1}, \rho_{2}, \cdots, \rho_{n}$ are the roots of the characteristic equation ordered according to decreasing absolute magnitude. Mark the two sets of points

$$
\epsilon_{1 j}, \epsilon_{2 j}, \cdots, \epsilon_{j-1, j}, 0 \text { and } \epsilon_{1 j}-1, \epsilon_{2 j}-1, \cdots, \epsilon_{j-1, j}-1,0
$$

and call them respectively

$$
P_{1}, P_{2}, \ldots, P_{j} \text { and } Q_{1}, Q_{2}, \ldots, Q_{j} \text {. }
$$

Construct the convex broken line $P_{1} P_{\sigma} \ldots P_{j}$ above which all the remaining points $P$ lie, and likewise construct the convex broken line $Q_{1} Q_{\theta} \cdots Q_{j}$ below which all the remaining points $Q$ lie. Let the acute angles which the successive sides of $P_{1} P_{\sigma} \cdots P_{j}$ and $Q_{1} Q_{\theta} \cdots Q_{j}$ make with the 
axis of reals be $\phi_{1}, \phi_{\sigma}, \ldots$ and $\psi_{1}, \psi_{\theta}, \ldots$ respectively. The critical rays between $\arg x=0$ and $\arg x=2 \pi$ are then

$$
\frac{\pi}{2}-\phi_{1}, \frac{\pi}{2}-\phi_{\sigma}, \cdots,-\frac{\pi}{2}+\psi_{\theta},-\frac{\pi}{2}+\psi_{1},
$$

in angular order. The asymptotic form of $g_{i j}(x)$ changes uniformly from

$$
s_{i a}(x) e^{2 \pi \lambda_{a j}{ }^{v-1 x}} c_{a j}^{(1)} \quad \text { to } \quad s_{i \beta}(x) e^{2 \pi \lambda_{\beta j} j^{v-1} x} c_{\beta j}^{(1))}
$$

along the critical ray $\arg x=\frac{1}{2} \pi-\phi_{a}$, and likewise from

$$
s_{i \gamma}(x) e^{2 \pi\left(\lambda_{\gamma j}-1\right)^{v-1 x}} e^{-2 \pi r \delta \delta^{\prime}-1} c_{\gamma j}^{(\mu-1)} \quad \text { to } \quad s_{i \delta}(x) e^{-2 \pi\left(r_{\delta j}-1\right)^{v-1} \bar{x}} e^{-2 \pi r_{\delta} \bar{y}^{-1}} c_{\delta j}^{(\mu-1)}
$$

along the critical ray $\arg x=-\frac{1}{2} \pi+\psi_{s}$. Between the last critical ray of the first set and the first of the last set the asymptotic form is given by $s_{i j}(x)$.

It is interesting to observe that with $g_{1 j}(x), \cdots, g_{n j}(x)$ there are associated at most $j-1$ critical rays in the first and in the fourth quadrants so that, for example, when $j=1$ there are no critical rays. But we have $g_{1}(x)=u_{1}(x)$, $\cdots, g_{n}(x)=u_{n}(x)$ and this result is in accord with Theorem 1 .

There are two special cases of exceptional interest. The first is that in which $\rho_{1}, \rho_{2}, \cdots, \rho_{n}$ are of equal absolute value. The quantities $\epsilon_{i j}$ are in this case all pure imaginaries and the broken lines $P_{1}, P_{\sigma}, \ldots$ and $Q_{1}, Q_{\theta}, \ldots$ reduce to segments of the imaginary axis, and we have $\phi_{1}=\pi / 2, \psi_{1}=\pi / 2$, so that the critical rays fall along the positive axis of reals, i. e., do not exist since this line is excluded throughout. In this event the elements of $G(x)$ preserve their asymptotic form throughout the entire plane, a result which agrees with that obtained in $\$ 1$. The second case is that in which the arguments of $\rho_{1}, \cdots, \rho_{n}$ are equal. In this case the quantities $\epsilon_{i j}$ are all real. In the first quadrant the only critical ray is the positive axis of imaginaries. In the fourth quadrant one finds critical rays different from the negative axis of imaginaries. How shall we reconcile these unsymmetric and apparently contradictory results, since by Theorem III the positive axis of imaginaries is not a critical ray? If one of the constants $c_{i j}^{(1)}$ which determine the change in asymptotic form vanishes, the formulas are still valid but do not give the dominant term.* The true critical rays in the first quadrant are symmetrically placed with respect to those in the fourth quadrant.

In general the effect of a zero value of $c_{i j}^{(1))}$ (or of $c_{i j}^{(\mu-1)}$ ) is to shift the actual change in asymptotic form to a secondary critical ray making a smaller angle with the positive axis of reals. The complete determination of its position may be effected when the constants $c_{i j}^{(l i)}$ are known.

The critical rays for these solutions have been determined by Galbrun by

* Just as, for example, if $u=x^{2}$ we have $u \sim e^{x}(0)$ along the positive axis of reals, but this does not give the dominant term. 
use of the Laplace transformation. It may be noted that the above results hold when the functions $a_{i j}(x)$ are only restricted as in (2), since only the form of the functions $p_{i j}(x)$ at $z=0$ and $z=\infty$ has been made use of. An interesting difference between the case when the functions $a_{i j}(x)$ are rational and the case when they are not lies in the fact that there are a limited number of possible positions for the secondary rays in the first case, and an unlimited number in the second.

\section{§ 7. The Fundamental Problem.}

The properties which we have thus far adduced completely characterise the solutions of a linear difference system (1) with rational coefficients, as is evidenced by the following theorem :

Theorem VI. Let $G(x)$ and $H(x)$ be two matrices of single-valued functions, analytic save for poles in the finite plane and such that their elements have the property

$\lim _{x=\infty} g_{i j}(x) x^{-\mu x} \rho_{j}^{-x} x^{-r_{j}}=s_{i j} \quad$ and $\quad \lim _{x=\infty} h_{i j}(x) x^{-\mu x} \rho_{j}^{-x} x^{-r_{j}}=s_{i j}, \quad\left(\left|s_{i j}\right| \neq 0\right)$

for $x$ in any left or right half plane rospectively, and furthermore let

$$
G(x)=H(x) P(x)
$$

where the elements of $P(x)$ are periodic of period 1 . Then $G(x)$ and $H(x)$ are the first and second principal matilix solutions of a system (4) in which the elements of $A(x)$ are rational in $x$, at least if $\rho_{1}, \rho_{2}, \ldots, \rho_{n}$ are distiuct.

Proof. If we put

$$
A(x)=G(x+1) G^{-1}(x)=H(x+1) H^{-1}(x),
$$

as we may by the hypothesis of the theorem, it is clear that the elements $a_{i j}(x)$ of $A(x)$ are single-valued and analytic in the finite plane except for poles. Furthermore, by direct computation, the elements $g_{i j}^{\prime}(x)$ and $h_{i j}^{\prime}(x)$ of $G^{-1}(x)$ and $H^{-1}(x)$ are seen to be such that

$$
\lim _{x=\infty} g_{i j}^{\prime}(x) x^{\mu x}\left(\rho_{i} e^{\mu}\right)^{r} \cdot x^{r_{i}}=\sigma_{i j} \quad \text { and } \quad \lim _{r=\infty} h_{i j}^{\prime}(x) \cdot c^{\delta \mu . r}\left(\rho_{i} e^{-\mu}\right)^{\delta . r} \cdot x^{r_{i}}=\sigma_{i j},
$$

in which $\sigma_{i j}$ denotes as before the element in the $i$ th row and $j$ th column of the matrix inverse to $\left(s_{i j}\right)$. The variable $x$ lies in any left and right half plane respectively. Therefore we have

$$
\begin{aligned}
a_{i j}(x) & =\sum_{\tau=1}^{n} g_{i \tau}(x+1) g_{\tau j}^{\prime}(x)=\sum_{\tau=1}^{n} h_{i \tau}(x+1) h_{\tau j}^{\prime}(x), \\
& =\sum_{\substack{\tau=1 \\
\text { Trans. Am. Math. Soc. } 19}}^{n}(x+1)^{\mu(x+1)}\left(\rho_{\tau} e^{-\mu}\right)^{r+1}(x+1)^{r} \tau\left(s_{i \tau}+\epsilon_{i \tau}\right) x^{-\mu . \tau}\left(\rho_{\tau} e^{-\mu}\right)^{-x} x^{-r} \tau\left(\sigma_{\tau j}+\epsilon_{1 j}^{\prime}\right), \\
&
\end{aligned}
$$


where $\epsilon_{i \tau}$ and $\epsilon_{\tau j}^{\prime}$ tend to zero for all large values of $x$, that is, in overlapping right and left half planes. This may be written

$$
a_{i j}(x)=x^{\mu} e^{-\mu}\left(1+\frac{1}{x}\right)^{\mu(x+1)} \sum_{\tau=1}^{n}\left(s_{i \tau} \rho_{\tau} \sigma_{\tau j}+\epsilon_{i \tau j}^{\prime \prime}\right),
$$

where $\epsilon_{i r j}^{\prime \prime}$ tends to zero as $x$ becomes infinite.

Thus $a_{i v}(x)$ must have a pole of order $\mu$ at $x=\infty$ with leading coefficient

$$
a_{i j}=\sum_{\tau=1}^{n} s_{i \tau} \rho_{\tau} \sigma_{\tau j}
$$

and is a rational function of $x$. In order to find the roots of the characteristic equation (3) in this case, we make an initial linear transformation with constant coefficients on $g_{1}(x), \cdots, g_{n}(x)$ in the linear difference system

$$
g_{i}(x)=\sum_{i=1}^{n} a_{i j}(x) g_{j}(x)
$$

which has been constructed. The constants $s_{i j}$ undergo the same transformation and may be reduced to $\delta_{i j}$; thus we shall have $a_{i j}=\delta_{i j} \rho_{j}$, so that the invariant characteristic equation is

$$
\left(\rho-\rho_{1}\right)\left(\rho-\rho_{2}\right) \cdots\left(\rho-\rho_{n}\right)=0
$$

with distinct roots $\rho_{1}, \ldots, \rho_{n}$ not zero. Hence the above system satisfies the restrictions of $\S 1$. According to the definition of principal matrix solutions, $G(x)$ and $H(x)$ must be the first and second principal matrix solutions, since the principal matrix solutions are the only ones which preserve a definite asymptotic form $s_{y}(x)$ even to terms of the first order in any left or right half plane respectively.

It is evident that the $n^{2}+2 n$ exponential constants $\rho_{i}, r_{i}, s_{i j}$ together with the functions $p_{v}(x)$ characterize the principal solutions more or less completely. Let us turn again to the case in which the coefficients $a_{4 j}(x)$ are polynomials of degree $\mu$ in $x$. In this case the equations (51) show that the functions $p_{v}(x)$ involves $n^{2} \mu-n$ further polynomial constants $\varepsilon_{y j}^{(l)}$. But among the exponential constants there are $n$ which are not essential since we can alter any set $s_{1 k}, \ldots, s_{n k}$ by an arbitrary multiplier and not affect the associated linear difference system (1). Hence there have been specified in all

$$
\left(n^{2}+2 n\right)+\left(n^{2} \mu-n\right)-n \quad \text { or } \quad n^{2}(\mu+1)
$$

characteristic constants counting only the ratios $s_{1 k}, s_{2 k}, \ldots, s_{n k}$ as characteristic constants. This number is precisely the number of arbitrary coefficients in the $n^{2}$ polynomials $a_{i y}(x)$, each of degree $\mu$ or less in $x$. Now the system (1) 
determines the constants, but not uniquely since $r_{1}, \ldots, r_{n}$ may be replaced by any determination $r_{1}+2 m_{1} \sqrt{-1}, \cdots, r_{n}+2 m_{n} \sqrt{-1}\left(m_{1}, \cdots, m_{n}\right.$ integers ) in the formal expansions. There is however exactly one set of characteristic constants for each such determination. If the characteristic constants are independent, one may conversely regard the coefficients in the polynomials $a_{i j}(x)$ as functions of the characteristic constants. The following definition and theorem go to show that conversely the characteristic constants do describe all the essenial properties of the solutions :

Definition. Two linear systems (1) are said to belong to the same class if one may be transformed into the other by a linear transformation with rational coefficients.

Theorem VII. Two linear difference systems (1) in which $\rho_{1}, \rho_{2}, \cdots, \rho_{n}$ are distinct and in which the functions $a_{i j}(x)$ are polynomials in $x$ of degree $\mu$ will belong to the same class if their characteristic constants are the same.

Proof. Let

$$
G_{1}(x+1)=A_{1}(x) G_{1}(x) \quad \text { and } \quad G_{2}(x+1)=A_{2}(x) G_{2}(x)
$$

be two systems of this kind, expressed in matrix notation, which possess the same characteristic constants. For convenience we suppose both of the equations transformed into a normal form in which

$$
a_{i j}(x)=\rho_{j}\left(\delta_{i j} x^{\mu}+a_{i j}^{(1)} x^{\mu-1}+\cdots\right) .
$$

This may always be effected by one and the same linear transformations with constant coefficients,

$$
G(x)=C \bar{G}(x),
$$

namely by that transformation which reduces the constants $s_{i j}$ to $\delta_{i j}$,

$$
\left(\delta_{i j}\right)=\left(c_{i j}\right)\left(s_{i j}\right)
$$

This is always possible by (7), and if one substitutes in the new formal expansions, it appears at once that the transformed elements of $A_{1}(x)$ and $A_{2}(x)$ have the form (55). The exponential constants $\rho_{j}, r_{j}$ and the polynomial constants are of course unaltered by this transformation, and we have explicitly in each case

$$
r_{i}=a_{i i}^{(1)}, \quad(i=1,2, \cdots, n) .
$$

This simplification being effected, let us consider the relation between the first principal matrix solutions $G_{1}(x)$ and $G_{2}(x)$. Write

We find then

$$
G_{1}(x)=R(x) G_{2}(x) \text {. }
$$

$$
R(x)=G_{1}(x) G_{2}^{-1}(x)=H_{1}(x) P(x)\left[H_{2}(x) P(x)\right]^{-1}=H_{1}(x) H_{2}^{-1}(x),
$$


where $H_{1}(x)$ and $H_{2}(x)$ are the second principal matrix solutions and $P(x)$ is the common matrix of fundamental periodic functions. It is now easy to demonstrate that $R(x)$ is a matrix of rational functions of $x$. The elements $a_{i j}(x)$ of this matrix have only poles in the finite plane and the relation (58) shows that

$$
\lim _{x=} R(x)=\delta_{i j}
$$

in the complete vicinis of $x=\infty$ on account of the common asymptotic form of $G_{1}$ and $G_{2}, H_{1}$ and $H_{2}$. In consequence of (59) the elements $r_{i j}(x)$ must be rational functions of $x$, analytic at $x=\infty$ and taking on the value $\delta_{i j}$ at that point.

Substitute the value of $G_{1}(x)$ given in $(57)$ in the first of the given difference systems. There results

$$
G_{2}(x+1)=\left[R^{-1}(x+1) A_{1}(x) R(x)\right] G_{2}(x) .
$$

A comparison with the second of the given systems shows then that

$$
A_{1}(x) R(x)=R(x+1) A_{2}(x) .
$$

This is the condition that the two systems belong to the same class and are transformable, the first into the second, by (57).

Even if this theorem is true there remains the possibility that not all of the characteristic constants are independent, although we are now certain that these constants effectively determine the transcendental properties of the solutions. We may however say something more of the dependence of solutions on the characteristic constants.

In the first place we observe that the exponential constants are expressed in terms of the coefficients in (56) when the reduction to normal form has been effected. Now the principal matrix solutions of (1) were constructed by means of certain limit determinants and a process of summation based on a contour integral. All the processes employed are uniformly convergent in the coefficients $a_{i j}^{(k)}$ and involve them analytically. It follows that the two principal solutions $G(x)$ and $H(x)$ of $(1)$ are analytic functions of the constants $a_{i j}^{\left(k_{i}\right)}$ as well as of $x$, and hence the elements of

$$
P(x)=G(x) H^{-1}(x)
$$

have the same property. This necessitates, of course, that the coefficients $c_{i j}^{(l)}$ be analytic in the coefficients $a_{i j}^{(l)}$. Let us take $\rho_{1}, \cdots, \rho_{n}, r_{1}, \ldots, r_{n}$ as fixed, which amounts to choosing $a_{i i}^{(1)}$ in (56) once for all. The coefficients $c_{i j}^{(l)}$ are then single-valued analytic functions of the remaining coefficients $a_{i j}^{(l)}$, which appear in precisely equal numbers.

Now only two things are possible: either the characteristic constants are independent and one may conversely regard the quantities $a_{i j}^{(\prime)}$ as analytic functions 
of the quantities $c_{i j}^{(l)}$, or else they are dependent. In this latter case we should be able to choose

$$
a_{i j}^{(k)}=\phi_{i j k}(\lambda) \quad \text { for all } i, j, k,
$$

where $\phi_{i j k}(\lambda)$ is an analytic function of the parameter $\lambda$, in such a way that the characteristic constants are independent of $\lambda$. The functions $a_{i j}(x)$ are then dependent on $\lambda$, so that we may write $A(x)=A(x, \lambda)$. Furthermore we can take generally

$$
A_{1}(x)=A\left(x, \lambda_{0}\right), \quad A_{2}(x)=A(x, \lambda)
$$

as two determinations of $A(x)$ which give equations (54) with the same characteristic constants, and we may take $\lambda=\lambda_{0}$ as a value of $\lambda$ for which the functions $\phi_{i j k}(x)$ are analytic. The matrix $R(x)$ is defined by (57), where $G_{1}(x)$ and $G_{2}(x)$ denote again the first principal solutions in (54). The elements $r_{i j}(x)$ are rational functions of $x$ by the preceding theorem.

We will now show that the functions $r_{i j}(x)$, which depend also on $\lambda$, must be polynomials in $x$. To this end we make the further assumption that no two of the $\mu^{n}$ roots of the determinant equation $\left|A\left(x, \lambda^{0}\right)\right|=0$ differ by integers. This is manifestly the general case.

If one of the elements of $R$ has a pole at a point, it. will be possible to form a group of such points

$$
\alpha, \alpha+1, \cdots, \beta
$$

containing the special point and such that $\alpha-1$ and $\beta+1$ are poles of no element of $R$. Now if we consider $(60)$ at $x=\beta$, we see that the right-hand member is analytic at this point, and that the left-hand nember will have at least one element with a pole at $x=\beta$ unless $\left|A_{1}(x)\right|=0$ at $x=\beta$. Hence we must have $A_{1}(\beta)=0$. Likewise if we consider the same equation at $x=\alpha-1$, we see that the left-hand member is analytic at this point, and that the right-hand member will have at least one element with a pole unless $\left|A_{2}(x)\right|=0$ at $x=\alpha-1$. Hence we must have $A_{2}(\alpha-1)=0$. But for $\lambda$ near to $\lambda_{0}$, the roots of

$$
\left|A_{1}(x)\right|=\left|A\left(x, \lambda_{0}\right)\right|=0 \quad \text { and } \quad\left|A_{2}(x)\right|=A(x, \lambda) \mid=0
$$

will nearly coincide and no two of them, as $\alpha-1$ and $\beta$ respectively, can be congruent and distinct on account of the hypothesis concerning the roots of $\left|A\left(x, \lambda_{0}\right)\right|=0$. It follows that the elements of $R(x)$ can have no poles in the finite plane, and hence are all polynomials in $x$. According to (59) these polynomials reduce to $\delta_{i j}$ at $x=\infty$, and hence are identically equal to $\delta_{i j}$. The elements of $A(x, \lambda)$ will not depend on the parameter $\lambda$, contrary to hypothesis. 
Hence the characteristic constants are independent, and the coefficients in the polynomials $a_{i j}(x)$ may be conversely regarded as analytic functions of these characteristic constants. A fundamental problem of the linear difference system is to study this functional relation and in particular to determine whether there exists $a$ system (1) with coefficients polynomial in $x$ of degree not greater than $\mu$ having any assigned set of characteristic constants.

Princeton Univergity. 